\title{
Nonlinear elliptic boundary value problem with equivalued surface in a domain with thin layer
}

\author{
Fengquan Li and Weilin Zou
}

\begin{abstract}
This paper deals with certain kinds of boundary value problems with equivalued surface of nonlinear elliptic equations on a domain with thin layer. We introduce the concept of renormalized solution to this problem. Existence and uniqueness of renormalized solutions are given, and the limit behaviour of solutions is studied in this paper.
\end{abstract}

Mathematics Subject Classification (2000). 35B37, 35B40, 49J20.

Keywords. Renormalized solution, Existence, Uniqueness, Equivalued surface, Equivalued interface, Nonlinear elliptic equations,

Limit behaviour of solutions.

\section{Introduction}

In many practical applications, especially in resistivity well-logging in petroleum exploitation, boundary value problems with equivalued surface are formulated in 1970s. It is a kind of nonlocal boundary value problems, which can also be used to give mathematical descriptions for some other problems in physics and mechanics. For this kind of boundary value problem, at least in linear case, a complete theory has been established (see $[8,9,11]$ ). In recent years some progress on the study of nonlinear boundary value problems with equivalued surface has been made (see $[5-7,11,12]$ ).

In resistivity well-logging, one may encounter a formation with crack domain, the resistivity of which is often difficult to be obtained. However, this crack domain is a thin layer compared with the whole formation (see [11]). In practical calculation, the variation of solutions near the thin layer should be quite large, and then in finite element procedure, it is necessary to have a refined partition of elements near the thin layer. This causes a complexity in 


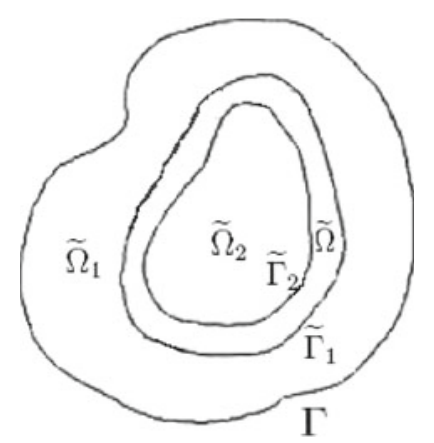

FIgURE 1 . The division of $\Omega$ with thin layer $\widetilde{\Omega}$

computation. In order to overcome this difficulty, when the thin layer is rather thin, the thin layer can be approximately regarded as an interface and correspondingly the boundary value problem with equivalued surface on the thin layer can be approximately replaced by the boundary value problem with equivalued interface. To prove the above conclusion, we need to study existence, uniqueness and limit behaviour of solutions for boundary value problems with equivalued surface in a domain with thin layer. The case of linear problems has been studied in [10]. In this paper, our main goal is to extend these results of [10] to nonlinear problems. For the sake of clarity in the exposition, we only consider the case that the thin layer $\tilde{\Omega}$ (see Fig. 1) is a good conductor (that is the potential $u$ should be a constant to be determined on $\tilde{\Omega}$ ), which is an important case in practical applications. Nevertheless, the methods involved in this paper can be applied to other cases in [10].

Here we consider the following nonlinear elliptic boundary value problems with equivalued surface on a domain with thin layer:

$$
\left(P_{1}\right) \begin{cases}-\sum_{i, j=1}^{N} \frac{\partial}{\partial x_{i}}\left(\tilde{a}_{i j}(x, u) \frac{\partial u}{\partial x_{j}}\right)=F(x) & \text { in } \widetilde{\Omega}_{1} \cup \widetilde{\Omega}_{2}, \\ u=0 & \text { on } \Gamma, \\ u=\tilde{C} \text { (a constant to be determined) } & \text { on } \widetilde{\Gamma}_{1} \cup \tilde{\Omega} \cup \widetilde{\Gamma}_{2}, \\ \int_{\widetilde{\Gamma}_{1}} \frac{\partial u}{\partial n_{L}} \mathrm{~d} s=\int_{\widetilde{\Gamma}_{2}} \frac{\partial u}{\partial n_{L}} \mathrm{~d} s+\tilde{A} \text { (a given constant), } & \end{cases}
$$

Let $\Omega$ be a bounded domain in $R^{N}(N \geq 2)$ with smooth outer boundary $\Gamma$ (see Fig. 1). Suppose that $\Omega$ is composed of three non-overlapping subdomains $\widetilde{\Omega}_{1}, \widetilde{\Omega}_{2}$ and $\widetilde{\Omega}$, and $\widetilde{\Gamma}_{1}$ and $\widetilde{\Gamma}_{2}$ are the interfaces of thin layer $\widetilde{\Omega}$ with $\widetilde{\Omega}_{1}$ and $\widetilde{\Omega}_{2}$ respectively. The unit outward normal vector $\vec{n}=\left(n_{1}, \ldots, n_{N}\right)$ takes the inward and outward directions (or vice versa) for the domain $\widetilde{\Omega}$ on $\widetilde{\Gamma}_{1}$ and $\widetilde{\Gamma}_{2}$.

$$
\frac{\partial u}{\partial n_{L}}=\sum_{i, j=1}^{N} \tilde{a}_{i j}(x, u) \frac{\partial u}{\partial x_{j}} n_{i}
$$

denotes the conormal derivative. 
In this paper we will discuss the existence, uniqueness and limit behaviour of renormalized solutions to problem $\left(P_{1}\right)$.

This paper is organized as follows: In Sect. 2, we will prove the existence and uniqueness of renormalized solutions to problems $\left(P_{1}\right)$. In Sect. 3 we will discuss nonlinear elliptic boundary value problem $(P)$ with equivalued interface. In Sect. 4 the limit behaviour of solutions to problems $\left(P_{1}\right)$ will be studied.

\section{Existence and uniqueness of renormalized solution to problem $\left(P_{1}\right)$}

In this section, we will discuss the existence and uniqueness of renormalized solution to problem $\left(P_{1}\right)$. We first make the following assumptions:

$\left(\widetilde{H}_{1}\right) \tilde{a}_{i j}(x, s)=\tilde{a}_{j i}(x, s)$, a.e. $x \in \Omega, \forall s \in R$. Moreover there exists a positive constant $\alpha$ such that for every $\xi=\left(\xi_{1}, \xi_{2}, \ldots, \xi_{N}\right) \in R^{N}$,

$$
\sum_{i, j=1}^{N} \tilde{a}_{i j}(x, s) \xi_{i} \xi_{j} \geq \alpha|\xi|^{2}, \quad \forall s \in R, \text { a.e. } x \in \Omega \text {. }
$$

$\left(\widetilde{H}_{2}\right)$ Let $\tilde{a}_{i j}: \Omega \times R \longrightarrow R$ be Carathéodory functions with $1 \leq i, j \leq N$. Assume that $\tilde{a}_{i j}(\cdot, 0) \in L^{\infty}(\Omega)$ and there exists a positive function $d \in L^{\infty}(\Omega)$ such that for every $s_{1}, s_{2} \in R$ and a.e. $x \in \Omega$,

$$
\left|\tilde{a}_{i j}\left(x, s_{1}\right)-\tilde{a}_{i j}\left(x, s_{2}\right)\right| \leq d(x)\left|s_{1}-s_{2}\right| .
$$

We also assume that $F \in L^{2}(\Omega)$.

Set

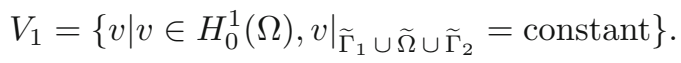

Definition 2.1. A measurable function $u \in V_{1}$ is called a weak solution to problem $\left(P_{1}\right)$, if $\tilde{a}_{i j}(x, u) D_{j} u \in L^{2}(\Omega)$ and

$$
\int_{\Omega} \sum_{i, j=1}^{N} \tilde{a}_{i j}(x, u) D_{j} u D_{i} v \mathrm{~d} x=\int_{\widetilde{\Omega}_{1} \cup \widetilde{\Omega}_{2}} F v \mathrm{~d} x+\left.\tilde{A} v\right|_{\widetilde{\Gamma}_{1} \cup \widetilde{\Omega}_{\cup} \cup \widetilde{\Gamma}_{2}}, \quad \forall v \in V_{1} .
$$

Remark 2.2. Under the above assumptions, it is impossible to obtain a $L^{\infty}$ estimate on weak solutions to problem $\left(P_{1}\right)$, moreover since $\tilde{a}_{i j}(x, u)$ is unbounded with respect to $u, \tilde{a}_{i j}(x, u) D_{j} u$ can not belong to $L^{2}(\Omega)$ and the term on the left hand side of (2.2) may not be well defined. Hence we are not able to prove the existence and uniqueness of weak solutions in the sense of Definition 2.1 to problem $\left(P_{1}\right)$. In order to overcome this difficulty, we will use the concept of renormalized solution introduced by Diperna and Lions in [3] for Boltzmann equations (see also $[1,2,7]$ ). The renormalized solution is different from the weak solution or the distribution solution because it is defined by truncation functions. 
Definition 2.3. A measurable function $u \in V_{1}$ is a renormalized solution to problem $\left(P_{1}\right)$, if for all $h(\cdot) \in W^{1, \infty}(R)$ with compact support and $\xi \in V_{1} \cap$ $L^{\infty}(\Omega), u$ satisfies

$$
\begin{aligned}
& \int_{\Omega} \sum_{i, j=1}^{N} \tilde{a}_{i j}(x, u) D_{j} u D_{i}(h(u) \xi) \mathrm{d} x \\
& \quad=\int_{\widetilde{\Omega}_{1} \cup \widetilde{\Omega}_{2}} F h(u) \xi \mathrm{d} x+\left.\tilde{A} h\left(\left.u\right|_{\widetilde{\Gamma}_{1} \cup \widetilde{\Omega} \cup \widetilde{\Gamma}_{2}}\right) \xi\right|_{\widetilde{\Gamma}_{1} \cup \widetilde{\Omega} \cup \widetilde{\Gamma}_{2}}
\end{aligned}
$$

and

$$
\lim _{m \rightarrow \infty} \int_{\{x \in \Omega: m \leq|u(x)| \leq m+1\}} \sum_{i, j=1}^{N} \tilde{a}_{i j}(x, u) D_{j} u D_{i} u \mathrm{~d} x=0 .
$$

For any given $k>0$, we defined the truncation function $T_{k}$ as follows:

$$
T_{k}(v)= \begin{cases}k & v>k \\ v & |v| \leq k \\ -k & v<-k\end{cases}
$$

Now we can state the existence and uniqueness result to problem $\left(P_{1}\right)$ as follows.

Theorem 2.4. Under hypotheses $\left(\widetilde{H}_{1}\right),\left(\widetilde{H}_{2}\right)$ and $F \in L^{2}(\Omega)$, problem $\left(P_{1}\right)$ admits a unique renormalized solution $u \in V_{1}$ in the sense of Definition 2.3.

Proof. (1) Proof of existence

Here we will use the method in [7]. We first consider the following problem:

$$
\left(P_{1 n}\right) \begin{cases}-\sum_{i, j=1}^{N} \frac{\partial}{\partial x_{i}}\left(\tilde{a}_{i j}\left(x, T_{n}\left(u_{n}\right)\right) \frac{\partial u_{n}}{\partial x_{j}}\right)=F(x) & \text { in } \widetilde{\Omega}_{1} \cup \widetilde{\Omega}_{2}, \\ u_{n}=0 & \text { on } \Gamma, \\ u_{n}=\tilde{C}_{n}(\text { a constant to be determined) } & \text { on } \widetilde{\Gamma}_{1} \cup \widetilde{\Omega} \cup \widetilde{\Gamma}_{2}, \\ \int_{\widetilde{\Gamma}_{1}} \frac{\partial u_{n}}{\partial n_{L}} \mathrm{~d} s=\int_{\widetilde{\Gamma}_{2}} \frac{\partial u_{n}}{\partial n_{L}} \mathrm{~d} s+\tilde{A} \text { (a given constant). } & \end{cases}
$$

Similarly to the first step in the proof of Theorem 3.1 in [12], using the pseudomonotone operator theory (cf. [13]), for any fixed $n>0$, problem $\left(P_{1 n}\right)$ admits a weak solution $u_{n} \in V_{1}$ such that

$$
\int_{\Omega} \sum_{i, j=1}^{N} \tilde{a}_{i j}\left(x, T_{n}\left(u_{n}\right)\right) D_{j} u_{n} D_{i} v \mathrm{~d} x=\int_{\widetilde{\Omega}_{1} \cup \widetilde{\Omega}_{2}} F v \mathrm{~d} x+\left.\tilde{A} v\right|_{\widetilde{\Gamma}_{1} \cup \widetilde{\Omega} \cup \widetilde{\Gamma}_{2}}, \quad \forall v \in V_{1} .
$$

Taking $v=u_{n}$ in $(2.3)$, by $\left(\widetilde{H}_{1}\right)$ and trace theorem, we get

$$
\alpha \int_{\Omega}\left|D u_{n}\right|^{2} \mathrm{~d} x \leq \int_{\widetilde{\Omega}_{1} \cup \widetilde{\Omega}_{2}} F u_{n} \mathrm{~d} x+C_{1}|\tilde{A}|\left\|u_{n}\right\|_{V_{1}},
$$

where $C_{1}$ is a positive constant independent of $n$. 
Hölder's inequality, Young's inequality and (2.4) imply that

$$
\int_{\Omega}\left|D u_{n}\right|^{2} \mathrm{~d} x \leq C_{2}\left(\|F\|_{L^{2}(\Omega)}^{2}+|\tilde{A}|^{2}\right),
$$

where $C_{2}$ is also a positive constant independent of $n$.

Inequality (2.5) implies that

$$
\left\|u_{n}\right\|_{V_{1}} \leq C_{3}\left(\|F\|_{L^{2}(\Omega)}+|\tilde{A}|\right),
$$

where $C_{3}$ is a positive constant independent of $n$.

By (2.6), there is a subsequence of $\left\{u_{n}\right\}$ (still denoted by $\left.\left\{u_{n}\right\}\right)$ such that

$$
u_{n} \rightarrow u \text { weakly in } V_{1} \text {. }
$$

Sobolev imbedding theorem and trace theorem yield

$$
u_{n} \rightarrow u \text { strongly in } L^{q}(\Omega)
$$

where

$$
\begin{cases}1 \leq q<\frac{2 N}{N-2}, & \text { if } N>2 \\ 1 \leq q<+\infty, & \text { if } N=2\end{cases}
$$

and

$$
\begin{aligned}
& \left.\left.u_{n}\right|_{\tilde{\Gamma}_{1} \cup \tilde{\Omega} \cup \tilde{\Gamma}_{2}} \rightarrow u\right|_{\widetilde{\Gamma}_{1} \cup \tilde{\Omega} \cup \widetilde{\Gamma}_{2}}, \\
& u_{n} \rightarrow u \text { a.e. in } \Omega .
\end{aligned}
$$

In the following, the proof will be divided into two steps.

Step 1. We will prove that for every $k>0, T_{k}\left(u_{n}\right)$ strongly converges to $T_{k}(u)$ in $V_{1}$.

For any fixed $k>0$, let $h>k$ and take $v=T_{2 k}\left(u_{n}-T_{h}\left(u_{n}\right)+T_{k}\left(u_{n}\right)-\right.$ $T_{k}(u)$ ) (introduced by Leone and Porretta in [4]) as a test function in (2.3). Setting $M=4 k+h$, it is easy to check that $D v=0$ where $\left|u_{n}\right|>M$, therefore for $n>M$, we can write $(2.3)$ as

$$
\int_{\Omega} \sum_{i, j=1}^{N} \tilde{a}_{i j}\left(x, T_{M}\left(u_{n}\right)\right) D_{j} u_{n} D_{i} v \mathrm{~d} x=\int_{\widetilde{\Omega}_{1} \cup \widetilde{\Omega}_{2}} F v \mathrm{~d} x+\left.\tilde{A} v\right|_{\widetilde{\Gamma}_{1} \cup \widetilde{\Omega}_{\tilde{\Gamma}} \widetilde{\Gamma}_{2}}, \quad \forall v \in V_{1} .
$$

Splitting the integral on the left side of (2.11) on the sets $\left\{x \in \Omega:\left|u_{n}(x)\right| \leq k\right\}$ and $\left\{x \in \Omega:\left|u_{n}(x)\right|>k\right\}$, by (2.1) we get 


$$
\begin{aligned}
& \int_{\Omega} \sum_{i, j=1}^{N} \tilde{a}_{i j}\left(x, T_{M}\left(u_{n}\right)\right) D_{j} u_{n} D_{i} T_{2 k}\left(u_{n}-T_{h}\left(u_{n}\right)+T_{k}\left(u_{n}\right)-T_{k}(u)\right) \mathrm{d} x \\
& =\int_{\left\{x \in \Omega:\left|u_{n}(x)\right| \leq k\right\}} \sum_{i, j=1}^{N} \tilde{a}_{i j}\left(x, T_{M}\left(u_{n}\right)\right) D_{j} u_{n} D_{i} T_{2 k}\left(u_{n}-T_{h}\left(u_{n}\right)+T_{k}\left(u_{n}\right)-T_{k}(u)\right) \mathrm{d} x \\
& \quad+\int_{\left\{x \in \Omega:\left|u_{n}(x)\right|>k\right\}} \sum_{i, j=1}^{N} \tilde{a}_{i j}\left(x, T_{M}\left(u_{n}\right)\right) D_{j} u_{n} D_{i} T_{2 k}\left(u_{n}-T_{h}\left(u_{n}\right)+T_{k}\left(u_{n}\right)-T_{k}(u)\right) \mathrm{d} x \\
& \geq \int_{\Omega} \sum_{i, j=1}^{N} \tilde{a}_{i j}\left(x, T_{k}\left(u_{n}\right)\right) D_{j} T_{k}\left(u_{n}\right) D_{i}\left(T_{k}\left(u_{n}\right)-T_{k}(u)\right) \mathrm{d} x \\
& \quad-\int_{\left\{x \in \Omega:\left|u_{n}(x)\right|>k\right\}} \sum_{i, j=1}^{N}\left|\tilde{a}_{i j}\left(x, T_{M}\left(u_{n}\right)\right)\right|\left|D_{j} u_{n}\right|\left|D_{i} T_{k}(u)\right| \mathrm{d} x .
\end{aligned}
$$

Relations (2.11) and (2.12) imply that

$$
\begin{aligned}
& \int_{\Omega} \sum_{i, j=1}^{N} \tilde{a}_{i j}\left(x, T_{k}\left(u_{n}\right)\right) D_{j}\left(T_{k}\left(u_{n}\right)-T_{k}(u)\right) D_{i}\left(T_{k}\left(u_{n}\right)-T_{k}(u)\right) \mathrm{d} x \\
& \leq \int_{\left\{x \in \Omega:\left|u_{n}(x)\right|>k\right\}} \sum_{i, j=1}^{N}\left|\tilde{a}_{i j}\left(x, T_{M}\left(u_{n}\right)\right)\right|\left|D_{j} u_{n}\right|\left|D_{i} T_{k}(u)\right| \mathrm{d} x \\
& \quad+\int_{\widetilde{\Omega}_{1} \cup \widetilde{\Omega}_{2}} F T_{2 k}\left(u_{n}-T_{h}\left(u_{n}\right)+T_{k}\left(u_{n}\right)-T_{k}(u)\right) \mathrm{d} x \\
& \quad+\left.\tilde{A} T_{2 k}\left(u_{n}-T_{h}\left(u_{n}\right)+T_{k}\left(u_{n}\right)-T_{k}(u)\right)\right|_{\widetilde{\Gamma}_{1} \cup \widetilde{\Omega} \cup \widetilde{\Gamma}_{2}} \\
& \quad-\int_{\Omega} \sum_{i, j=1}^{N} \tilde{a}_{i j}\left(x, T_{k}\left(u_{n}\right)\right) D_{j} T_{k}(u) D_{i}\left(T_{k}\left(u_{n}\right)-T_{k}(u)\right) \mathrm{d} x \\
& =I_{1}+I_{2}+I_{3}-I_{4} .
\end{aligned}
$$

For any fixed $h>0,\left(\widetilde{H}_{2}\right)$ and $(2.5)$ imply that $\left|\tilde{a}_{i j}\left(\cdot, T_{M}\left(u_{n}\right)\right)\right|\left|D_{j}\left(u_{n}\right)\right|$ is bounded in $L^{2}(\Omega)$, while $\chi_{\left\{\left|u_{n}\right|>k\right\}}\left|D_{i} T_{k}(u)\right|$ converges strongly to zero in $L^{2}(\Omega)$ as $n$ tends to the infinity, hence we have

$$
\lim _{n \rightarrow \infty} I_{1}=\lim _{n \rightarrow \infty} \int_{\Omega} \sum_{i, j=1}^{N}\left|\tilde{a}_{i j}\left(x, T_{M}\left(u_{n}\right)\right)\right|\left|D_{j}\left(u_{n}\right)\right|\left|D_{i} T_{k}(u)\right| \chi_{\left\{\left|u_{n}\right|>k\right\}} \mathrm{d} x=0 .
$$

Relation (2.10) and Lebesgue's dominated convergence theorem imply that

$$
\lim _{n \rightarrow \infty} I_{2}=\int_{\widetilde{\Omega}_{1} \cup \widetilde{\Omega}_{2}} F T_{2 k}\left(u-T_{h}(u)\right) \mathrm{d} x .
$$

We derive from (2.9) that

$$
\lim _{n \rightarrow \infty} I_{3}=\tilde{A} T_{2 k}\left(\left.u\right|_{\widetilde{\Gamma}_{1} \cup \widetilde{\Omega} \cup \widetilde{\Gamma}_{2}}-T_{h}\left(\left.u\right|_{\widetilde{\Gamma}_{1} \cup \widetilde{\Omega} \cup \widetilde{\Gamma}_{2}}\right)\right) .
$$

By $\left(\widetilde{H}_{2}\right),(2.10)$ and Lebesgue's dominated convergence theorem, we have

$$
\tilde{a}_{i j}\left(\cdot, T_{k}\left(u_{n}\right)\right) D_{j} T_{k}(u) \rightarrow \tilde{a}_{i j}\left(\cdot, T_{k}(u)\right) D_{j} T_{k}(u) \text { strongly in } L^{2}(\Omega) .
$$


Thus, from (2.7) and (2.17) it follows that

$$
\lim _{n \rightarrow \infty} I_{4}=0 \text {. }
$$

Let $n$ tend to the infinity in (2.13). We derive from (2.1), (2.13)-(2.16) and (2.18) that

$$
\begin{aligned}
& \varlimsup_{n \rightarrow \infty} \alpha \int_{\Omega}\left|D T_{k}\left(u_{n}\right)-D T_{k}(u)\right|^{2} \\
& \leq \int_{\widetilde{\Omega}_{1} \cup \widetilde{\Omega}_{2}} F T_{2 k}\left(u-T_{h}(u)\right) \mathrm{d} x \\
& \quad+\tilde{A} T_{2 k}\left(\left.u\right|_{\widetilde{\Gamma}_{1} \cup \widetilde{\Omega} \cup \widetilde{\Gamma}_{2}}-T_{h}\left(\left.u\right|_{\widetilde{\Gamma}_{1} \cup \widetilde{\Omega} \cup \widetilde{\Gamma}_{2}}\right)\right) .
\end{aligned}
$$

Let $h \rightarrow \infty$ in (2.19), we get

$$
\varlimsup_{n \rightarrow \infty} \alpha \int_{\Omega}\left|D T_{k}\left(u_{n}\right)-D T_{k}(u)\right|^{2} \leq 0
$$

which implies that

$$
D T_{k}\left(u_{n}\right) \rightarrow D T_{k}(u) \text { in } L^{2}(\Omega), \quad \text { as } n \rightarrow \infty .
$$

Step 2. We will prove that $u$ is a renormalized solution to problem $\left(P_{1}\right)$. For any given $\xi \in V_{1} \cap L^{\infty}(\Omega)$ and $h(\cdot) \in W^{1, \infty}(R)$ with compact support belonging to $[-k, k]$, taking $v=h\left(u_{n}\right) \xi$ in $(2.3)$, we get

$$
\begin{aligned}
& \int_{\Omega} \sum_{i, j=1}^{N} \tilde{a}_{i j}\left(x, T_{n}\left(u_{n}\right)\right) D_{j} u_{n} D_{i}\left(h\left(u_{n}\right) \xi\right) \mathrm{d} x \\
& \quad=\int_{\widetilde{\Omega}_{1} \cup \widetilde{\Omega}_{2}} F h\left(u_{n}\right) \xi \mathrm{d} x+\left.\tilde{A} h\left(\left.u_{n}\right|_{\widetilde{\Gamma}_{1} \cup \widetilde{\Omega} \cup \widetilde{\Gamma}_{2}}\right) \xi\right|_{\widetilde{\Gamma}_{1} \cup \widetilde{\Omega} \cup \widetilde{\Gamma}_{2}} \cdot
\end{aligned}
$$

For any given $n>k$, we have

$$
\begin{aligned}
& \int_{\Omega} \sum_{i, j=1}^{N} \tilde{a}_{i j}\left(x, T_{n}\left(u_{n}\right)\right) D_{j} u_{n} D_{i}\left(h\left(u_{n}\right) \xi\right) \mathrm{d} x \\
& =\int_{\Omega} \sum_{i, j=1}^{N} \tilde{a}_{i j}\left(x, T_{k}\left(u_{n}\right)\right) D_{j} T_{k}\left(u_{n}\right) D_{i}\left(h\left(u_{n}\right) \xi\right) \mathrm{d} x \\
& =\int_{\Omega} \sum_{i, j=1}^{N} \tilde{a}_{i j}\left(x, T_{k}\left(u_{n}\right)\right) D_{j} T_{k}\left(u_{n}\right) D_{i} T_{k}\left(u_{n}\right) h^{\prime}\left(u_{n}\right) \xi \mathrm{d} x \\
& \quad+\int_{\Omega} \sum_{i, j=1}^{N} \tilde{a}_{i j}\left(x, T_{k}\left(u_{n}\right)\right) D_{j} T_{k}\left(u_{n}\right) D_{i} \xi h\left(u_{n}\right) \mathrm{d} x \\
& =J_{1}+J_{2} .
\end{aligned}
$$

By $\left(\widetilde{H}_{2}\right),(2.10)$ and $(2.20)$ we get

$$
\lim _{n \rightarrow \infty} J_{1}=\int_{\Omega} \sum_{i, j=1}^{N} \tilde{a}_{i j}\left(x, T_{k}(u)\right) D_{j} T_{k}(u) D_{i} T_{k}(u) h^{\prime}(u) \xi \mathrm{d} x
$$


and

$$
\lim _{n \rightarrow \infty} J_{2}=\int_{\Omega} \sum_{i, j=1}^{N} \tilde{a}_{i j}\left(x, T_{k}(u)\right) D_{j} T_{k}(u) D_{i} \xi h(u) \mathrm{d} x .
$$

Let $n \rightarrow \infty$ in (2.21), it follows from (2.8)-(2.10), (2.22)-(2.24) that

$$
\int_{\Omega} \sum_{i, j=1}^{N} \tilde{a}_{i j}(x, u) D_{j} u D_{i}(h(u) \xi) \mathrm{d} x=\int_{\tilde{\Omega}_{1} \cup \tilde{\Omega}_{2}} F h(u) \xi \mathrm{d} x+\left.\tilde{A} h\left(\left.u\right|_{\tilde{\Gamma}_{1} \cup \tilde{\Omega} \cup \tilde{\Gamma}_{2}}\right) \xi\right|_{\widetilde{\Gamma}_{1} \cup \tilde{\Omega} \cup \tilde{\Gamma}_{2}} .
$$

For any given $m>0$, taking $v=T_{m+1}\left(u_{n}\right)-T_{m}\left(u_{n}\right)$ in $(2.3)$, we get

$$
\begin{aligned}
& \int_{\Omega} \sum_{i, j=1}^{N} \tilde{a}_{i j}\left(x, T_{n}\left(u_{n}\right)\right) D_{j} u_{n} D_{i}\left[T_{m+1}\left(u_{n}\right)-T_{m}\left(u_{n}\right)\right] \mathrm{d} x \\
& =\int_{\widetilde{\Omega}_{1} \cup \widetilde{\Omega}_{2}} F\left[T_{m+1}\left(u_{n}\right)-T_{m}\left(u_{n}\right)\right] \mathrm{d} x \\
& \quad+\tilde{A}\left[T_{m+1}\left(\left.u_{n}\right|_{\widetilde{\Gamma}_{1} \cup \widetilde{\Omega} \cup \widetilde{\Gamma}_{2}}\right)-T_{m}\left(\left.u_{n}\right|_{\widetilde{\Gamma}_{1} \cup \widetilde{\Omega} \cup \widetilde{\Gamma}_{2}}\right)\right] .
\end{aligned}
$$

For $n>m+1$, we get

$$
\begin{aligned}
& \int_{\left\{x \in \Omega: m \leq\left|u_{n}(x)\right| \leq m+1\right\}} \sum_{i, j=1}^{N} \tilde{a}_{i j}\left(x, u_{n}\right) D_{j} u_{n} D_{i} u_{n} \mathrm{~d} x \\
& =\int_{\Omega} \sum_{i, j=1}^{N} \tilde{a}_{i j}\left(x, T_{n}\left(u_{n}\right)\right) D_{j} u_{n} D_{i}\left[T_{m+1}\left(u_{n}\right)-T_{m}\left(u_{n}\right)\right] \mathrm{d} x \\
& =\int_{\widetilde{\Omega}_{1} \cup \widetilde{\Omega}_{2}} F\left[T_{m+1}\left(u_{n}\right)-T_{m}\left(u_{n}\right)\right] \mathrm{d} x \\
& \quad+\widetilde{A}\left[T_{m+1}\left(\left.u_{n}\right|_{\widetilde{\Gamma}_{1} \cup \widetilde{\Omega} \cup \widetilde{\Gamma}_{2}}\right)-T_{m}\left(\left.u_{n}\right|_{\widetilde{\Gamma}_{1} \cup \widetilde{\Omega} \cup \widetilde{\Gamma}_{2}}\right)\right] .
\end{aligned}
$$

However

$$
\begin{aligned}
& \int_{\left\{x \in \Omega: m \leq\left|u_{n}(x)\right| \leq m+1\right\}} \sum_{i, j=1}^{N} \tilde{a}_{i j}\left(x, u_{n}\right) D_{j} u_{n} D_{i} u_{n} \mathrm{~d} x \\
& =\int_{\Omega} \sum_{i, j=1}^{N} \tilde{a}_{i j}\left(x, T_{m+1}\left(u_{n}\right)\right) D_{j} T_{m+1}\left(u_{n}\right) \\
& \quad \times D_{i} T_{m+1}\left(u_{n}\right) \chi_{\left\{x \in \Omega: m \leq\left|u_{n}(x)\right| \leq m+1\right\}} \mathrm{d} x .
\end{aligned}
$$


By $\left(\widetilde{H}_{2}\right),(2.10)$ and replacing $k$ by $m+1$ in $(2.20)$, we get

$$
\begin{aligned}
& \int_{\Omega} \sum_{i, j=1}^{N} \tilde{a}_{i j}\left(x, T_{m+1}\left(u_{n}\right)\right) D_{j} T_{m+1}\left(u_{n}\right) D_{i} T_{m+1}\left(u_{n}\right) \chi_{\left\{x \in \Omega: m \leq\left|u_{n}(x)\right| \leq m+1\right\}} \mathrm{d} x \\
& \longrightarrow \int_{\Omega} \sum_{i, j=1}^{N} \tilde{a}_{i j}\left(x, T_{m+1}(u)\right) D_{j} T_{m+1}(u) D_{i} T_{m+1}(u) \chi_{\{x \in \Omega: m \leq|u(x)| \leq m+1\}} \mathrm{d} x \\
& =\int_{\{x \in \Omega: m \leq|u(x)| \leq m+1\}} \sum_{i, j=1}^{N} \tilde{a}_{i j}(x, u) D_{j} u D_{i} u \mathrm{~d} x .
\end{aligned}
$$

Let $n$ tend to the infinity in (2.26). We conclude from (2.8)-(2.10) and (2.27), (2.28) that

$$
\begin{aligned}
& \int_{\{x \in \Omega: m \leq|u(x)| \leq m+1\}} \sum_{i, j=1}^{N} \tilde{a}_{i j}(x, u) D_{j} u D_{i} u \mathrm{~d} x \\
& =\int_{\widetilde{\Omega}_{1} \cup \widetilde{\Omega}_{2}} F\left[T_{m+1}(u)-T_{m}(u)\right] \mathrm{d} x \\
& \quad+\tilde{A}\left[T_{m+1}\left(\left.u\right|_{\widetilde{\Gamma}_{1} \cup \widetilde{\Omega} \cup \widetilde{\Gamma}_{2}}\right)-T_{m}\left(\left.u\right|_{\widetilde{\Gamma}_{1} \cup \widetilde{\Omega} \cup \widetilde{\Gamma}_{2}}\right)\right] .
\end{aligned}
$$

We conclude from (2.29) and Lebesgue's dominated convergence theorem that

$$
\lim _{m \rightarrow \infty} \int_{\{x \in \Omega: m \leq|u(x)| \leq m+1\}} \sum_{i, j=1}^{N} \tilde{a}_{i j}(x, u) D_{j} u D_{i} u \mathrm{~d} x=0 .
$$

From (2.25) and (2.30), we can deduce that $u$ is a renormalized solution to problem $\left(P_{1}\right)$.

(2) Proof of uniqueness

Here the idea of this proof comes from [1]. Let $u_{1}, u_{2} \in V_{1}$ be two renormalized solutions to problem $\left(P_{1}\right)$ in the sense of Definition 2.3. Then for all $h(\cdot) \in W^{1, \infty}(R)$ with compact support and $\xi \in V_{1} \cap L^{\infty}(\Omega)$, we have

$$
\begin{aligned}
& \int_{\Omega} \sum_{i, j=1}^{N} \tilde{a}_{i j}\left(x, u_{s}\right) D_{j} u_{s} D_{i}\left(h\left(u_{s}\right) \xi\right) \mathrm{d} x \\
& \quad=\int_{\widetilde{\Omega}_{1} \cup \widetilde{\Omega}_{2}} F h\left(u_{s}\right) \xi \mathrm{d} x+\left.\tilde{A} h\left(\left.u_{s}\right|_{\tilde{\Gamma}_{1} \cup \tilde{\Omega} \cup \tilde{\Gamma}_{2}}\right) \xi\right|_{\tilde{\Gamma}_{1} \cup \tilde{\Omega} \cup \tilde{\Gamma}_{2}}
\end{aligned}
$$

and

$$
\lim _{m \rightarrow \infty} \int_{\left\{x \in \Omega: m \leq\left|u_{s}(x)\right| \leq m+1\right\}} \sum_{i, j=1}^{N} \tilde{a}_{i j}\left(x, u_{s}\right) D_{j} u_{s} D_{i} u_{s} \mathrm{~d} x=0, \quad s=1,2 .
$$

Let $h_{n}(\cdot) \in W^{1, \infty}(R)$ with compact support be defined as follows

$$
h_{n}(t)=1-\left|T_{n+1}(t)-T_{n}(t)\right|, \quad \forall t \in R .
$$


For any given $0 \leq k<1$ and $n>0$, taking $h(\cdot)=h_{n}(\cdot), \xi=T_{k}\left(u_{1}-u_{2}\right)$ in (2.31), we get

$$
\begin{aligned}
\int_{\Omega} & \sum_{i, j=1}^{N}\left[\tilde{a}_{i j}\left(x, u_{1}\right) D_{j} u_{1} h_{n}\left(u_{1}\right)-\tilde{a}_{i j}\left(x, u_{2}\right) D_{j} u_{2} h_{n}\left(u_{2}\right)\right] D_{i} T_{k}\left(u_{1}-u_{2}\right) \mathrm{d} x \\
= & \int_{\Omega} \sum_{i, j=1}^{N} \tilde{a}_{i j}\left(x, u_{2}\right) D_{j} u_{2} D_{i} u_{2} h_{n}^{\prime}\left(u_{2}\right) T_{k}\left(u_{1}-u_{2}\right) \mathrm{d} x \\
& -\int_{\Omega} \sum_{i, j=1}^{N} \tilde{a}_{i j}\left(x, u_{1}\right) D_{j} u_{1} D_{i} u_{1} h_{n}^{\prime}\left(u_{1}\right) T_{k}\left(u_{1}-u_{2}\right) \mathrm{d} x \\
& +\int_{\widetilde{\Omega}_{1} \cup \widetilde{\Omega}_{2}} F\left[h_{n}\left(u_{1}\right)-h_{n}\left(u_{2}\right)\right] T_{k}\left(u_{1}-u_{2}\right) \mathrm{d} x \\
& +\tilde{A}\left[h_{n}\left(\left.u_{1}\right|_{\tilde{\Gamma}_{1} \cup \tilde{\Omega} \cup \tilde{\Gamma}_{2}}\right)-h_{n}\left(\left.u_{2}\right|_{\tilde{\Gamma}_{1} \cup \tilde{\Omega} \cup \tilde{\Gamma}_{2}}\right)\right] T_{k}\left(\left.\left(u_{1}-u_{2}\right)\right|_{\tilde{\Gamma}_{1} \cup \tilde{\Omega} \cup \tilde{\Gamma}_{2}}\right) \\
= & K_{1}-K_{2}+K_{3}+K_{4} .
\end{aligned}
$$

The left term in (2.34) can be written as

$$
\begin{aligned}
\int_{\Omega} & \sum_{i, j=1}^{N}\left[\tilde{a}_{i j}\left(x, u_{1}\right) D_{j} u_{1} h_{n}\left(u_{1}\right)-\tilde{a}_{i j}\left(x, u_{2}\right) D_{j} u_{2} h_{n}\left(u_{2}\right)\right] D_{i} T_{k}\left(u_{1}-u_{2}\right) \mathrm{d} x \\
= & \frac{1}{2} \int_{\Omega} \sum_{i, j=1}^{N}\left[h_{n}\left(u_{1}\right)+h_{n}\left(u_{2}\right)\right]\left[\tilde{a}_{i j}\left(x, u_{1}\right) D_{j} u_{1}-\tilde{a}_{i j}\left(x, u_{2}\right) D_{j} u_{2}\right] \\
& \times D_{i} T_{k}\left(u_{1}-u_{2}\right) \mathrm{d} x \\
& +\frac{1}{2} \int_{\Omega} \sum_{i, j=1}^{N}\left[h_{n}\left(u_{1}\right)-h_{n}\left(u_{2}\right)\right]\left[\tilde{a}_{i j}\left(x, u_{1}\right) D_{j} u_{1}+\tilde{a}_{i j}\left(x, u_{2}\right) D_{j} u_{2}\right] \\
& \times D_{i} T_{k}\left(u_{1}-u_{2}\right) \mathrm{d} x \\
= & A(n, k)+B(n, k) .
\end{aligned}
$$

Writing $A(n, k)$ as

$$
\begin{aligned}
A(n, k)= & \frac{1}{4} \int_{\Omega} \sum_{i, j=1}^{N}\left[h_{n}\left(u_{1}\right)+h_{n}\left(u_{2}\right)\right]\left[\tilde{a}_{i j}\left(x, u_{1}\right)-\tilde{a}_{i j}\left(x, u_{2}\right)\right] \\
& \times D_{j}\left(u_{1}+u_{2}\right) D_{i} T_{k}\left(u_{1}-u_{2}\right) \mathrm{d} x \\
& +\frac{1}{4} \int_{\Omega} \sum_{i, j=1}^{N}\left[h_{n}\left(u_{1}\right)+h_{n}\left(u_{2}\right)\right]\left[\tilde{a}_{i j}\left(x, u_{1}\right)+\tilde{a}_{i j}\left(x, u_{2}\right)\right] \\
& \times D_{j}\left(u_{1}-u_{2}\right) D_{i} T_{k}\left(u_{1}-u_{2}\right) \mathrm{d} x \\
= & C(n, k)+D(n, k) .
\end{aligned}
$$


Noting (2.33), we have

$$
\begin{aligned}
\left|K_{1}\right| & \leq k \int_{\Omega} \sum_{i, j=1}^{N}\left|h_{n}^{\prime}\left(u_{2}\right)\right| \tilde{a}_{i j}\left(x, u_{2}\right) D_{j} u_{2} D_{i} u_{2} \mathrm{~d} x \\
& =k \int_{\left\{x \in \Omega: n \leq\left|u_{2}(x)\right| \leq n+1\right\}} \sum_{i, j=1}^{N} \tilde{a}_{i j}\left(x, u_{2}\right) D_{j} u_{2} D_{i} u_{2} \mathrm{~d} x
\end{aligned}
$$

and

$$
\begin{aligned}
\left|K_{2}\right| & \leq k \int_{\Omega} \sum_{i, j=1}^{N}\left|h_{n}^{\prime}\left(u_{1}\right)\right| \tilde{a}_{i j}\left(x, u_{1}\right) D_{j} u_{1} D_{i} u_{1} \mathrm{~d} x \\
& =k \int_{\left\{x \in \Omega: n \leq\left|u_{1}(x)\right| \leq n+1\right\}} \sum_{i, j=1}^{N} \tilde{a}_{i j}\left(x, u_{1}\right) D_{j} u_{1} D_{i} u_{1} \mathrm{~d} x .
\end{aligned}
$$

From (2.34)-(2.38), we have

$$
\begin{aligned}
D(n, k) \leq & k \int_{\left\{x \in \Omega: n \leq\left|u_{1}(x)\right| \leq n+1\right\}} \sum_{i, j=1}^{N} \tilde{a}_{i j}\left(x, u_{1}\right) D_{j} u_{1} D_{i} u_{1} \mathrm{~d} x \\
& +k \int_{\left\{x \in \Omega: n \leq\left|u_{2}(x)\right| \leq n+1\right\}} \sum_{i, j=1}^{N} \tilde{a}_{i j}\left(x, u_{2}\right) D_{j} u_{2} D_{i} u_{2} \mathrm{~d} x \\
& +K_{3}+K_{4}+|B(n, k)|+|C(n, k)| .
\end{aligned}
$$

For any given $y, z \in R^{N}$, by $\left(\widetilde{H}_{1}\right)$, it is easy to get the following inequality

$$
\left|\sum_{i, j=1}^{N} \tilde{a}_{i j}(x, s) y_{i} z_{j}\right| \leq\left(\sum_{i, j=1}^{N} \tilde{a}_{i j}(x, s) y_{i} y_{j}\right)^{1 / 2}\left(\sum_{i, j=1}^{N} \tilde{a}_{i j}(x, s) z_{i} z_{j}\right)^{1 / 2}
$$

For any given $\varepsilon>0,(2.39)$ and Young's inequality yield

$$
\begin{aligned}
|B(n, k)| \leq & \frac{\varepsilon}{2} \int_{\Omega} \sum_{i, j=1}^{N}\left[h_{n}\left(u_{1}\right)+h_{n}\left(u_{2}\right)\right]\left[\tilde{a}_{i j}\left(x, u_{1}\right)+\tilde{a}_{i j}\left(x, u_{2}\right)\right] \\
& \times D_{j} T_{k}\left(u_{1}-u_{2}\right) D_{i} T_{k}\left(u_{1}-u_{2}\right) \mathrm{d} x \\
& +\frac{1}{2 \varepsilon} \int_{\left\{\left|u_{1}-u_{2}\right|<k\right\}} \sum_{i, j=1}^{N}\left|h_{n}\left(u_{1}\right)-h_{n}\left(u_{2}\right)\right| \\
& \times\left[\tilde{a}_{i j}\left(x, u_{1}\right) D_{j} u_{1} D_{i} u_{1}+\tilde{a}_{i j}\left(x, u_{2}\right) D_{j} u_{2} D_{i} u_{2}\right] \mathrm{d} x .
\end{aligned}
$$


By Hölder's inequality, Young's inequality and $\left(\widetilde{H}_{1}\right)-\left(\widetilde{H}_{2}\right)$, we find that

$$
\begin{aligned}
|C(n, k)| \leq & \frac{1}{4} \int_{\left\{0<\left|u_{1}-u_{2}\right|<k\right\}} \sum_{i, j=1}^{N}\left[h_{n}\left(u_{1}\right)+h_{n}\left(u_{2}\right)\right] d(x)\left|u_{1}-u_{2}\right| \\
& \times\left|D_{j}\left(u_{1}+u_{2}\right)\right|\left|D_{i} T_{k}\left(u_{1}-u_{2}\right)\right| \mathrm{d} x \\
\leq & \frac{k}{4} \int_{\left\{0<\left|u_{1}-u_{2}\right|<k\right\}} \sum_{i, j=1}^{N}\left[h_{n}\left(u_{1}\right)+h_{n}\left(u_{2}\right)\right]\|d\|_{L^{\infty}(\Omega)} \\
& \times\left|D_{j}\left(u_{1}+u_{2}\right)\right|\left|D_{i} T_{k}\left(u_{1}-u_{2}\right)\right| \mathrm{d} x \\
\leq & \frac{k^{2}}{4 \varepsilon}\|d\|_{L^{\infty}(\Omega)}^{2} \int_{\left\{0<\left|u_{1}-u_{2}\right|<k\right\}}\left[h_{n}\left(u_{1}\right)+h_{n}\left(u_{2}\right)\right]\left(\left|D u_{1}\right|^{2}+\left|D u_{2}\right|^{2}\right) \mathrm{d} x \\
& +\frac{\varepsilon}{4} \int_{\left\{0<\left|u_{1}-u_{2}\right|<k\right\}}\left[h_{n}\left(u_{1}\right)+h_{n}\left(u_{2}\right)\right]\left|D T_{k}\left(u_{1}-u_{2}\right)\right|^{2} \mathrm{~d} x \\
\leq & \frac{k^{2}}{4 \varepsilon}\|d\|_{L^{\infty}(\Omega)}^{2} \int_{\left\{0<\left|u_{1}-u_{2}\right|<k\right\}}\left[h_{n}\left(u_{1}\right)+h_{n}\left(u_{2}\right)\right]\left(\left|D u_{1}\right|^{2}+\left|D u_{2}\right|^{2}\right) \mathrm{d} x \\
& +\frac{\varepsilon}{8 \alpha} \int_{\Omega} \sum_{i, j=1}^{N}\left[h_{n}\left(u_{1}\right)+h_{n}\left(u_{2}\right)\right]\left[\tilde{a}_{i j}\left(x, u_{1}\right)+\tilde{a}_{i j}\left(x, u_{2}\right)\right] \\
& \times D_{j} T_{k}\left(u_{1}-u_{2}\right) D_{i} T_{k}\left(u_{1}-u_{2}\right) \mathrm{d} x .
\end{aligned}
$$

Taking $\varepsilon=\frac{\alpha}{4 \alpha+1}$ in (2.40) and (2.41), we get

$$
\begin{aligned}
\int_{\Omega} \sum_{i, j=1}^{N}\left[h_{n}\left(u_{1}\right)+h_{n}\left(u_{2}\right)\right]\left[\tilde{a}_{i j}\left(x, u_{1}\right)+\tilde{a}_{i j}\left(x, u_{2}\right)\right] \\
\quad \times D_{j} T_{k}\left(u_{1}-u_{2}\right) D_{i} T_{k}\left(u_{1}-u_{2}\right) \mathrm{d} x \\
\leq C_{4}\left\{k \int_{\left\{x \in \Omega: n \leq\left|u_{1}(x)\right| \leq n+1\right\}} \sum_{i, j=1}^{N} \tilde{a}_{i j}\left(x, u_{1}\right) D_{j} u_{1} D_{i} u_{1} \mathrm{~d} x\right. \\
\quad+k \int_{\left\{x \in \Omega: n \leq\left|u_{2}(x)\right| \leq n+1\right\}} \sum_{i, j=1}^{N} \tilde{a}_{i j}\left(x, u_{2}\right) D_{j} u_{2} D_{i} u_{2} \mathrm{~d} x \\
\quad+\int_{\left\{0<\left|u_{1}-u_{2}\right|<k\right\}} \sum_{i, j=1}^{N}\left|h_{n}\left(u_{1}\right)-h_{n}\left(u_{2}\right)\right| \\
\quad \times\left[\tilde{a}_{i j}\left(x, u_{1}\right) D_{j} u_{1} D_{i} u_{1}+\tilde{a}_{i j}\left(x, u_{2}\right) D_{j} u_{2} D_{i} u_{2}\right] \mathrm{d} x \\
\quad+K_{3}+K_{4}+k^{2}\|d\|_{L}^{2}(\Omega) \\
\left.\quad \times \int_{0<\left|u_{1}-u_{2}\right|<k}\left[h_{n}\left(u_{1}\right)+h_{n}\left(u_{2}\right)\right]\left(\left|D u_{1}\right|^{2}+\left|D u_{2}\right|^{2}\right) \mathrm{d} x\right\}
\end{aligned}
$$

where $C_{4}$ is a positive constant independent of $n$. 
From (2.33), it follows that $\left|h_{n}^{\prime}(t)\right|=1$ for $n \leq|t| \leq n+1$ and $h_{n}^{\prime}(t)=0$ for $|t| \geq n+1$ or $|t| \leq n$. Furthermore we have $h_{n}\left(u_{1}\right)-h_{n}\left(u_{2}\right)=h_{n}^{\prime}(\theta)\left(u_{1}-u_{2}\right)$ where $\theta$ is between $u_{1}$ and $u_{2}$. Let $0 \leq k<1$, then

$$
\begin{aligned}
& \int_{\left\{0<\left|u_{1}-u_{2}\right|<k\right\}} \sum_{i, j=1}^{N}\left|h_{n}\left(u_{1}\right)-h_{n}\left(u_{2}\right)\right|\left[\tilde{a}_{i j}\left(x, u_{1}\right) D_{j} u_{1} D_{i} u_{1}+\tilde{a}_{i j}\left(x, u_{2}\right)\right. \\
& \left.\quad \times D_{j} u_{2} D_{i} u_{2}\right] \mathrm{d} x \\
& \leq \int_{\left\{0<\left|u_{1}-u_{2}\right|<k\right\} \cap\left\{n-1 \leq\left|u_{1}\right| \leq n+2\right\} \cap\left\{n-1 \leq\left|u_{2}\right| \leq n+2 \mid\right\}} \\
& \quad \times k \sum_{i, j=1}^{N}\left[\tilde{a}_{i j}\left(x, u_{1}\right) D_{j} u_{1} D_{i} u_{1}+\tilde{a}_{i j}\left(x, u_{2}\right) D_{j} u_{2} D_{i} u_{2}\right] \mathrm{d} x
\end{aligned}
$$

By (2.32) and (2.43), we find that

$$
\begin{aligned}
& \lim _{n \rightarrow \infty} \int_{\left\{0<\left|u_{1}-u_{2}\right|<k\right\}} \sum_{i, j=1}^{N}\left|h_{n}\left(u_{1}\right)-h_{n}\left(u_{2}\right)\right| \\
& \quad \times\left[\tilde{a}_{i j}\left(x, u_{1}\right) D_{j} u_{1} D_{i} u_{1}+\tilde{a}_{i j}\left(x, u_{2}\right) D_{j} u_{2} D_{i} u_{2}\right] \mathrm{d} x=0 .
\end{aligned}
$$

Since

$$
h_{n}\left(u_{s}\right) \longrightarrow 1 \text { a.e. } x \in \Omega, \quad s=1,2
$$

and

$$
h_{n}\left(u_{s}\right) \stackrel{\text { weak }^{*}}{\longrightarrow} 1 \text { in } L^{\infty}(\Omega)
$$

we have

$$
\lim _{n \rightarrow \infty} K_{3}=\lim _{n \rightarrow \infty} \int_{\widetilde{\Omega}_{1} \cup \widetilde{\Omega}_{2}} F\left[h_{n}\left(u_{1}\right)-h_{n}\left(u_{2}\right)\right] T_{k}\left(u_{1}-u_{2}\right) \mathrm{d} x=0,
$$

and

$$
\begin{aligned}
& \lim _{n \rightarrow \infty} K_{4}=\lim _{n \rightarrow \infty} \tilde{A}\left[h_{n}\left(\left.u_{1}\right|_{\tilde{\Gamma}_{1} \cup \tilde{\Omega} \cup \tilde{\Gamma}_{2}}\right)-h_{n}\left(\left.u_{2}\right|_{\tilde{\Gamma}_{1} \cup \tilde{\Omega} \cup \tilde{\Gamma}_{2}}\right)\right] \\
& \quad \times T_{k}\left(\left.\left(u_{1}-u_{2}\right)\right|_{\widetilde{\Gamma}_{1} \cup \tilde{\Omega} \cup \tilde{\Gamma}_{2}}\right) \mathrm{d} x=0 .
\end{aligned}
$$

Let $n \rightarrow \infty$ in (2.42). Assumption $\left(\widetilde{H}_{1}\right),(2.32),(2.42)-(2.44)$ and $(2.47)-(2.48)$ imply that

$$
\int_{\Omega}\left|D T_{k}\left(u_{1}-u_{2}\right)\right|^{2} \mathrm{~d} x \leq C_{5} k^{2} \int_{\left\{0<\left|u_{1}-u_{2}\right|<k\right\}}\left|D u_{1}\right|^{2}+\left|D u_{2}\right|^{2} \mathrm{~d} x,
$$

where $C_{5}$ is a positive constant independent of $n$ and $k$.

Inequality (2.49) and the absolute continuity of integral imply that

$$
\lim _{k \rightarrow 0} \frac{1}{k^{2}} \int_{\Omega}\left|D T_{k}\left(u_{1}-u_{2}\right)\right|^{2} \mathrm{~d} x=0 .
$$


Setting $E_{k}=\left\{x \in \Omega:\left|u_{1}-u_{2}\right|>k\right\}, \Omega_{1}=\left\{x \in \Omega: u_{1}(x) \neq u_{2}(x)\right\},(2.50)$ and Poincaré's inequality yield

$$
\text { meas } \begin{aligned}
E_{k} & =\frac{1}{k^{2}} \int_{E_{k}} k^{2} \mathrm{~d} x \leq \frac{1}{k^{2}} \int_{\Omega}\left|T_{k}\left(u_{1}-u_{2}\right)\right|^{2} \mathrm{~d} x \\
& \leq C_{6} \frac{1}{k^{2}} \int_{\Omega}\left|D T_{k}\left(u_{1}-u_{2}\right)\right|^{2} \mathrm{~d} x \longrightarrow 0, \quad \text { as } k \rightarrow 0,
\end{aligned}
$$

where $C_{6}$ is a positive constant independent of $k$.

From (2.51) it follows meas $\Omega_{1}=0$. Thus $u_{1}(x)=u_{2}(x)$, a.e. $x \in \Omega$.

Remark 2.5. In [7] the author has discussed the existence and uniqueness of renormalized solution nonlinear elliptic boundary value problem with equivalued surface but without Dirichlet boundary conditions and thin layer. Furthermore, we remark that the zero-order term in the equation is crucial to the proof of uniqueness of renormalized solution (see Theorem 3.1 in [7]). Here the equation in problem $\left(P_{1}\right)$ has not zero-order term. Thus we do not use the same approach as that of [7] to the proof of uniqueness.

Similarly to Theorem 4.1 in [7], it is easy to prove the following theorem.

Theorem 2.6. (i) Assume that $u \in V_{1}$ and $a_{i j}(\cdot, u) D_{j} u \in L^{2}(\Omega), i, j=$ $1,2, \ldots, N$. Then $u$ is a weak solution to problem $\left(P_{1}\right)$ if and only if $u$ is a renormalized solution to problem $\left(P_{1}\right)$.

(ii) If $u \in V_{1} \cap L^{\infty}(\Omega)$, then $u$ is a weak solution to problem $\left(P_{1}\right)$ if and only if $u$ is a renormalized solution to problem $\left(P_{1}\right)$.

\section{Nonlinear elliptic boundary value problem with equivalued interface}

In order to study the limit behaviour of solutions to problem $\left(P_{1}\right)$, we need to study the following equivalued interface problem $(P)$. Here we give another division of $\Omega$ as shown in Fig. 2 . $\Omega$ is composed of two non-overlapping subdomains $\Omega_{1}$ and $\Omega_{2}$, and $\widetilde{\Gamma}$ is the interface of $\Omega_{1}$ and $\Omega_{2}$.

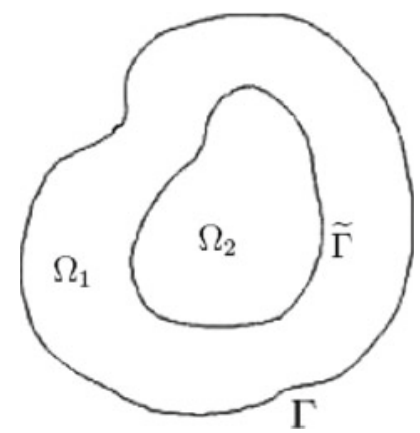

Figure 2. The division of $\Omega$ with an interface $\widetilde{\Gamma}$ 
In this section we will consider the following nonlinear elliptic boundary value problem with equivalued interface:

$(P) \begin{cases}-\sum_{i, j=1}^{N} \frac{\partial}{\partial x_{i}}\left(a_{i j}(x, u) \frac{\partial u}{\partial x_{j}}\right)=F(x) & \text { in } \Omega_{1} \cup \Omega_{2}, \\ u=0 & \text { on } \Gamma, \\ u_{+}=u_{-}=C(\text { a constant to be determined }) & \text { on } \widetilde{\Gamma}, \\ \int_{\widetilde{\Gamma}}\left(\frac{\partial u}{\partial n_{L}}\right)_{+} \mathrm{d} s=\int_{\widetilde{\Gamma}}\left(\frac{\partial u}{\partial n_{L}}\right)_{-} \mathrm{d} s+A \text { (a given constant), }\end{cases}$

where the subscripts + and - denote the values on both sides of $\widetilde{\Gamma}$.

We make the following assumptions:

$\left(H_{1}\right) a_{i j}(x, s)=a_{j i}(x, s)$, a.e. $x \in \Omega, \forall s \in R$. There exists a positive constant $\alpha$ such that for every $\xi=\left(\xi_{1}, \xi_{2}, \ldots, \xi_{N}\right) \in R^{N}$,

$$
\sum_{i, j=1}^{N} a_{i j}(x, s) \xi_{i} \xi_{j} \geq \alpha|\xi|^{2}, \quad \forall s \in R, \text { a.e. } x \in \Omega
$$

$\left(H_{2}\right)$ Let $a_{i j}: \Omega \times R \longrightarrow R$ be Carathéodory functions with $1 \leq i, j \leq N$. Assume that $a_{i j}(\cdot, 0) \in L^{\infty}(\Omega)$ and there exists a positive function $d \in$ $L^{\infty}(\Omega)$ such that for every $s_{1}, s_{2} \in R$ and a.e. $x \in \Omega$,

$$
\left|a_{i j}\left(x, s_{1}\right)-a_{i j}\left(x, s_{2}\right)\right| \leq d(x)\left|s_{1}-s_{2}\right|(i, j=1,2, \ldots, N) .
$$

Let

$$
V=\left\{v\left|v \in H_{0}^{1}(\Omega), v\right|_{\widetilde{\Gamma}}=\text { constant }\right\}
$$

Definition 3.1. A measurable function $u \in V$ is a renormalized solutions to problem $(P)$, if for all $h(\cdot) \in W^{1, \infty}(R)$ with compact support and $\xi \in V \cap$ $L^{\infty}(\Omega)$, u satisfies

$$
\int_{\Omega} \sum_{i, j=1}^{N} a_{i j}(x, u) D_{j} u D_{i}(h(u) \xi) \mathrm{d} x=\int_{\Omega} F h(u) \xi \mathrm{d} x+\left.A h\left(\left.u\right|_{\widetilde{\Gamma}}\right) \xi\right|_{\widetilde{\Gamma}}
$$

and

$$
\lim _{m \rightarrow \infty} \int_{\{x \in \Omega: m \leq|u(x)| \leq m+1\}} \sum_{i, j=1}^{N} a_{i j}(x, u) D_{j} u D_{i} u \mathrm{~d} x=0 .
$$

Theorem 3.2. Under hypotheses $\left(H_{1}\right),\left(H_{2}\right)$ and $F \in L^{2}(\Omega)$, problem $(P)$ admits a unique renormalized solution $u \in V$ in the sense of Definition 3.1.

Proof. The proof of this theorem is similar to Theorem 2.4, thus we omit the details. 


\section{Limit behaviour of solutions to problem $\left(P_{1}\right)$}

In this section, we will study the limit behaviour of solutions to boundary value problem $\left(P_{1}\right)$. More precisely, let $\varepsilon>0$ be a small parameter. Replace $\widetilde{\Omega}_{1}, \widetilde{\Omega}_{2}, \widetilde{\Omega}$ by $\Omega_{1}^{\varepsilon}, \Omega_{2}^{\varepsilon}, \widetilde{\Omega}^{\varepsilon}$, and interfaces $\widetilde{\Gamma}_{1}$ and $\widetilde{\Gamma}_{2}$ by interfaces $\widetilde{\Gamma}_{1}^{\varepsilon}$ and $\widetilde{\Gamma}_{2}^{\varepsilon}$, respectively as shown in Fig. 3 .

Here we will discuss the following problem

$$
\left(P_{\varepsilon}\right) \begin{cases}-\sum_{i, j=1}^{N} \frac{\partial}{\partial x_{i}}\left(a_{i j}^{\varepsilon}\left(x, u_{\varepsilon}\right) \frac{\partial u_{\varepsilon}}{\partial x_{j}}\right)=F(x) & \text { in } \Omega_{1}^{\varepsilon} \cup \Omega_{2}^{\varepsilon}, \\ u_{\varepsilon}=0 & \text { on } \Gamma, \\ u_{\varepsilon}=\widetilde{C}_{\varepsilon}(\text { a constant to be determined }) & \text { on } \widetilde{\Gamma}_{1}^{\varepsilon} \cup \widetilde{\Omega}^{\varepsilon} \cup \widetilde{\Gamma}_{2}^{\varepsilon}, \\ \int_{\widetilde{\Gamma}_{1}^{\varepsilon}} \frac{\partial u_{\varepsilon}}{\partial n_{L^{\varepsilon}}} \mathrm{d} s=\int_{\widetilde{\Gamma}_{2}^{\varepsilon}} \frac{\partial u_{\varepsilon}}{\partial n_{L^{\varepsilon}}} \mathrm{d} s+A_{\varepsilon} \text { (a given constant). } & \end{cases}
$$

We make the following assumptions:

$\left(H_{3}\right) \widetilde{\Gamma} \subset \widetilde{\Omega}^{\varepsilon}, \forall \varepsilon>0 ; \widetilde{\Omega}^{\varepsilon}$ shrinks to $\Gamma$ as $\varepsilon \rightarrow 0$.

$\left(H_{4}\right)$ For any given domain $\widetilde{\Omega}$ such that $\widetilde{\Gamma} \subset \widetilde{\Omega} \subset \Omega$, then for any $\varepsilon>0$ small enough, we have

$$
\tilde{\Omega}^{\varepsilon} \subset \widetilde{\Omega} .
$$

$\left(H_{5}\right)$ For all $\varepsilon>0, a_{i j}^{\varepsilon}(x, s)=a_{j i}^{\varepsilon}(x, s)$, a.e. $x \in \Omega, \forall s \in R$. There exists a positive constant $\alpha$ independent of $\varepsilon$ such that

$$
\sum_{i, j=1}^{N} a_{i j}^{\varepsilon}\left(x, u_{\varepsilon}\right) \xi_{i} \xi_{j} \geq \alpha|\xi|^{2}, \forall \xi=\left(\xi_{1}, \xi_{2}, \cdots, \xi_{N}\right) \in R^{N}, \quad \forall x \in \Omega .
$$

$\left(H_{6}\right)$ Let $a_{i j}^{\varepsilon}: \Omega \times R \longrightarrow R$ be Carathéodory functions with $1 \leq i, j \leq N$. We also assume that there exists a positive constant $\beta$ independent of $\varepsilon$ such that $\left\|a_{i j}^{\varepsilon}(\cdot, 0)\right\|_{L^{\infty}(\Omega)} \leq \beta$. Moreover there exists a positive function $d \in L^{\infty}(\Omega)$ such that for every $s_{1}, s_{2} \in R$ and a.e. $x \in \Omega$,

$$
\left|a_{i j}^{\varepsilon}\left(x, s_{1}\right)-a_{i j}^{\varepsilon}\left(x, s_{2}\right)\right| \leq d(x)\left|s_{1}-s_{2}\right|, \quad \forall s_{1}, s_{2} \in R \text {, a.e. } x \in \Omega
$$

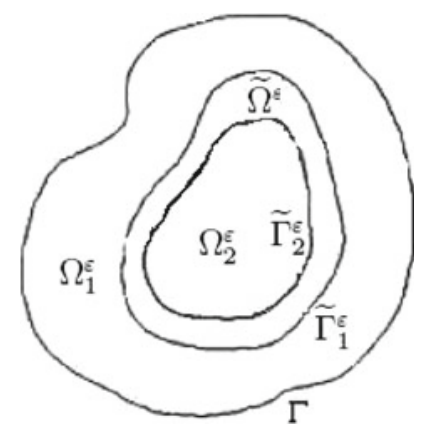

FiguRE 3. The division of $\Omega$ with thin layer described by parameter $\varepsilon$ 
$\left(H_{7}\right)$ For any given domain $\widetilde{\Omega}$ such that $\widetilde{\Gamma} \subset \widetilde{\Omega} \subset \Omega$, as $\varepsilon \rightarrow 0$

$$
a_{i j}^{\varepsilon}(x, s) \rightarrow a_{i j}(x, s) \quad \text { strongly in } L^{\infty}(\Omega \backslash \widetilde{\Omega}), \quad \forall s \in R .
$$

Set

$$
V_{\varepsilon}=\left\{v\left|v \in H_{0}^{1}(\Omega), v\right|_{\widetilde{\Gamma}_{1}^{\varepsilon} \cup \widetilde{\Omega}^{\varepsilon} \cup \widetilde{\Gamma}_{2}^{\varepsilon}}=\text { constant }\right\} .
$$

Definition 4.1. A measurable function $u_{\varepsilon} \in V_{\varepsilon}$ is a renormalized solutions to problem $\left(P_{\varepsilon}\right)$, if for all $h \in W^{1, \infty}(R)$ with compact support and $\xi \in V_{\varepsilon} \cap$ $L^{\infty}(\Omega), u$ satisfies

$$
\begin{aligned}
& \int_{\Omega} \sum_{i, j=1}^{N} a_{i j}^{\varepsilon}\left(x, u_{\varepsilon}\right) D_{j} u_{\varepsilon} D_{i}\left(h\left(u_{\varepsilon}\right) \xi\right) \mathrm{d} x \\
& \quad=\int_{\Omega_{1}^{\varepsilon} \cup \Omega_{2}^{\varepsilon}} F h\left(u_{\varepsilon}\right) \xi \mathrm{d} x+\left.A_{\varepsilon} h\left(\left.u_{\varepsilon}\right|_{\tilde{\Gamma}_{1}^{\varepsilon} \cup \tilde{\Omega}^{\varepsilon} \cup \tilde{\Gamma}_{2}^{\varepsilon}}\right) \xi\right|_{\tilde{\Gamma}_{1}^{\varepsilon} \cup \tilde{\Omega}^{\varepsilon} \cup \tilde{\Gamma}_{2}^{\varepsilon}}
\end{aligned}
$$

and

$$
\lim _{m \rightarrow \infty} \int_{\left\{x \in \Omega: m \leq\left|u_{\varepsilon}(x)\right| \leq m+1\right\}} \sum_{i, j=1}^{N} a_{i j}^{\varepsilon}\left(x, u_{\varepsilon}\right) D_{j} u_{\varepsilon} D_{i} u_{\varepsilon} \mathrm{d} x=0 .
$$

Remark 4.2. For every fixed $\varepsilon>0$, if $\left(H_{5}\right)-\left(H_{6}\right)$ and $F \in L^{2}(\Omega)$ hold, we can similarly prove that problem $\left(P_{\varepsilon}\right)$ admits a unique solution $u_{\varepsilon} \in V_{\varepsilon}$ in the sense of Definition 4.1.

Now we give the limit behaviour of solutions to problem $\left(P_{\varepsilon}\right)$ as follows.

Theorem 4.3. Suppose that $\left(H_{1}\right)-\left(H_{7}\right)$ and $F \in L^{2}(\Omega)$ hold. If $\varepsilon \rightarrow 0, A_{\varepsilon} \rightarrow A$, then for every renormalized solution $u_{\varepsilon}$ to problem $\left(P_{\varepsilon}\right)$ we have

$$
u_{\varepsilon} \longrightarrow u \text { weakly in } V \text {, }
$$

where $u$ is the renormalized solution to problem $(P)$ and $V$ is as in (3.1).

In order to prove Theorem 4.3, we need the following lemma.

Lemma 4.4. Under hypotheses $\left(H_{3}\right)$ and $\left(H_{4}\right)$, for any given $\varphi \in V$, there exist $\varphi_{\varepsilon} \in V_{\varepsilon}$ such that as $\varepsilon \longrightarrow 0$,

$$
\varphi_{\varepsilon} \longrightarrow \varphi \quad \text { strongly in } V \text {, }
$$

where $V$ is as in (3.1).

Proof. For convenience, we may assume that the origin is an interior point of $\Omega_{2}$ (see Fig. 2).

For fixed $\varepsilon>0$ small enough, let $\Omega_{2}^{\varepsilon}=\left\{x(1-\varepsilon) \mid x \in \Omega_{2}\right\}, \quad \Omega_{1}^{\prime}=\left\{\frac{x}{1-\varepsilon} \mid x \in \Omega_{2}\right\}, \quad \Omega_{1}^{\varepsilon}=\Omega \backslash \Omega_{1}^{\prime}, \quad \widetilde{\Omega}^{\varepsilon}=\Omega_{1}^{\prime} \backslash \Omega_{2}^{\varepsilon}$.

Define $\Gamma^{\varepsilon}=\{x(1-\varepsilon) \mid x \in \Gamma\}$ and assume that $\widetilde{\Gamma}_{1}^{\varepsilon}, \widetilde{\Gamma}_{2}^{\varepsilon}$ are the interfaces of $\widetilde{\Omega}^{\varepsilon}$ with $\Omega_{1}^{\varepsilon}$ and $\Omega_{2}^{\varepsilon}$ (see Fig. 4). 


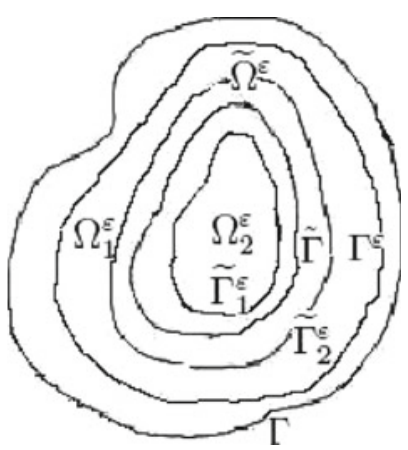

Figure 4. Scaling down or up the division of $\Omega$ in Fig. 2

We define $\psi_{\varepsilon}$ as follows:

$$
\psi_{\varepsilon}=\psi_{\varepsilon}^{+}-\psi_{\varepsilon}^{-}
$$

where

$$
\begin{aligned}
& \psi_{\varepsilon}^{+}= \begin{cases}\left(\psi((1-\varepsilon) x)-\sup _{\Gamma^{\varepsilon}} \psi^{+}(x)\right)^{+} & x \in \Omega_{1}^{\varepsilon}, \\
\left(\left.\psi(x)\right|_{\widetilde{\Gamma}}-\sup _{\Gamma^{\varepsilon}} \psi^{+}(x)\right)^{+} & x \in \widetilde{\Gamma}_{1}^{\varepsilon} \cup \widetilde{\Omega}^{\varepsilon} \cup \widetilde{\Gamma}_{2}^{\varepsilon}, \\
\left(\psi\left(\frac{x}{1-\varepsilon}\right)-\sup _{\Gamma^{\varepsilon}} \psi^{+}(x)\right)^{+} & x \in \Omega_{2}^{\varepsilon},\end{cases} \\
& \psi_{\varepsilon}^{-}= \begin{cases}\left(\psi((1-\varepsilon) x)-\inf _{\Gamma^{\varepsilon}} \psi^{-}(x)\right)^{-} & x \in \Omega_{1}^{\varepsilon}, \\
\left(\left.\psi(x)\right|_{\tilde{\Gamma}}-\inf _{\Gamma^{\varepsilon}} \psi^{-}(x)\right)^{-} & x \in \widetilde{\Gamma}_{1}^{\varepsilon} \cup \widetilde{\Omega}^{\varepsilon} \cup \widetilde{\Gamma}_{2}^{\varepsilon}, \\
\left(\psi\left(\frac{x}{1-\varepsilon}\right)-\inf _{\Gamma^{\varepsilon}} \psi^{-}(x)\right)^{-} & x \in \Omega_{2}^{\varepsilon} .\end{cases}
\end{aligned}
$$

It is easy to see that $\psi_{\varepsilon}^{+} \in V_{\varepsilon}, \psi_{\varepsilon}^{-} \in V_{\varepsilon}$. Thus we have $\psi_{\varepsilon} \in V_{\varepsilon}$. We can easily prove that $\varphi_{\varepsilon}^{+}$and $\varphi_{\varepsilon}^{-}$strongly converge to $\varphi^{+}$and $\varphi^{-}$in $V$ respectively. Hence $\varphi_{\varepsilon}$ strongly converges to $\varphi$. We omit the details.

Proof of Theorem 4.3. For any given $\varepsilon>0$, problem $\left(P_{\varepsilon}\right)$ admits a unique renormalized solution $u_{\varepsilon} \in V_{\varepsilon}$ by Theorem 2.4 and there exists a sequence $\left\{u_{\varepsilon_{n}}\right\} \subset V_{\varepsilon}$ such that

$$
u_{\varepsilon_{n}} \longrightarrow u_{\varepsilon} \text { weakly in } V_{\varepsilon}, \quad \text { as } n \rightarrow \infty
$$

and

$$
\begin{aligned}
& \int_{\Omega} \sum_{i, j=1}^{N} a_{i j}^{\varepsilon}\left(x, T_{n}\left(u_{\varepsilon_{n}}\right)\right) D_{j} u_{\varepsilon_{n}} D_{i} v \mathrm{~d} x \\
& \quad=\int_{\Omega_{1}^{\varepsilon} \cup \Omega_{2}^{\varepsilon}} F v \mathrm{~d} x+\left.A_{\varepsilon} v\right|_{\tilde{\Gamma}_{1}^{\varepsilon \cup \Omega^{\varepsilon} \cup \tilde{\Gamma}_{2}^{\varepsilon}}}, \quad \forall v \in V_{\varepsilon} .
\end{aligned}
$$


By virtue of $v \in V_{\varepsilon}$ and $\left(H_{3}\right),\left.v\right|_{\widetilde{\Gamma}_{1}^{\varepsilon} \cup \tilde{\Omega}^{\varepsilon} \cup \tilde{\Gamma}_{2}^{\varepsilon}}=\left.v\right|_{\widetilde{\Gamma}}$. Thus, we can write (4.4) as follows:

$$
\int_{\Omega} \sum_{i, j=1}^{N} a_{i j}^{\varepsilon}\left(x, T_{n}\left(u_{\varepsilon_{n}}\right)\right) D_{j} u_{\varepsilon_{n}} D_{i} v \mathrm{~d} x=\int_{\Omega_{1}^{\varepsilon} \cup \Omega_{2}^{\varepsilon}} F v \mathrm{~d} x+\left.A_{\varepsilon} v\right|_{\tilde{\Gamma}}, \quad \forall v \in V_{\varepsilon} .
$$

Take $v=u_{\varepsilon_{n}}$ as a test function in (4.5). From $\left(H_{5}\right)$, Sobolev imbedding theorem, trace theorem, Poincaré's inequality and Young's inequality, it follows

$$
\left\|u_{\varepsilon_{n}}\right\|_{V_{\varepsilon}} \leq C_{7}\left[\|F\|_{L^{2}(\Omega)}+A_{\varepsilon}\right]
$$

where $C_{7}$ is a positive constant depending on $\alpha$, meas $\widetilde{\Gamma}$ and meas $\Omega$, independent of $\varepsilon, n$.

Relations (4.3) and (4.6) imply that

$$
\left\|u_{\varepsilon}\right\|_{V_{\varepsilon}} \leq C_{7}\left[\|F\|_{L^{2}(\Omega)}+A_{\varepsilon}\right]
$$

Hence there exists a positive constant $C_{8}$ independent of $\varepsilon$ such that

$$
\left\|u_{\varepsilon}\right\|_{V} \leq C_{8} .
$$

Thus there is a subsequence of $\left\{u_{\varepsilon}\right\}$ (still denote by $\left\{u_{\varepsilon}\right\}$ ) such that

$$
u_{\varepsilon} \longrightarrow u \text { weakly in } V, \text { as } \varepsilon \rightarrow 0 \text {. }
$$

Sobolev imbedding theorem, trace theorem and $\left(H_{3}\right)$ yield

$$
u_{\varepsilon} \rightarrow u \text { strongly in } L^{q}(\Omega), \quad \text { as } \varepsilon \rightarrow 0,
$$

where

$$
\begin{cases}1 \leq q<\frac{2 N}{N-2}, & N>2 \\ 1 \leq q<+\infty, & N=2\end{cases}
$$

and

$$
\begin{aligned}
& \left.u_{\varepsilon}\right|_{\tilde{\Gamma}_{1}^{\varepsilon} \cup \tilde{\Omega}^{\varepsilon} \cup \tilde{\Gamma}_{\tilde{2}}^{\varepsilon}}=\left.\left.u_{\varepsilon}\right|_{\tilde{\Gamma}} \rightarrow u\right|_{\tilde{\Gamma}}, \\
& u_{\varepsilon} \rightarrow u \text { a.e in } \Omega .
\end{aligned}
$$

In the following, the proof will be divided into three steps.

Step 1: We will prove that for any given $k>0$,

$$
T_{k}\left(u_{\varepsilon}\right) \rightarrow T_{k}(u) \quad \text { strongly in } V, \quad \text { as } \varepsilon \rightarrow 0 .
$$

By Lemma 4.4, there is a sequence $\omega_{\varepsilon_{0}} \in V_{\varepsilon_{0}}$ such that

$$
\omega_{\varepsilon_{0}} \rightarrow u \quad \text { strongly in } V, \quad \text { as } \varepsilon_{0} \rightarrow 0 .
$$

Hence for any given $k>0$, we have

$$
T_{k}\left(\omega_{\varepsilon_{0}}\right) \rightarrow T_{k}(u) \text { strongly in } V, \quad \text { as } \varepsilon_{0} \rightarrow 0 .
$$

Fixed $\varepsilon_{0}>0$, by $\left(H_{4}\right)$, for any $\varepsilon$ with $0<\varepsilon<\varepsilon_{0}$

$$
\widetilde{\Omega}^{\varepsilon} \subset \widetilde{\Omega}^{\varepsilon_{0}} \quad \text { and } \quad \omega_{\varepsilon_{0}} \in V_{\varepsilon} .
$$


Let $h>k>0$, taking $\xi=\xi_{\varepsilon}=T_{2 k}\left(u_{\varepsilon}-T_{h}\left(u_{\varepsilon}\right)+T_{k}\left(u_{\varepsilon}\right)-T_{k}\left(\omega_{\varepsilon_{0}}\right)\right)$ and $h(t)=h_{n}(t)$ in (4.1), where $h_{n}(t)$ is defined in (2.33), we get

$$
\begin{aligned}
& \int_{\Omega} \sum_{i, j=1}^{N} a_{i j}^{\varepsilon}\left(x, u_{\varepsilon}\right) D_{j} u_{\varepsilon} D_{i} \xi_{\varepsilon} h_{n}\left(u_{\varepsilon}\right) \mathrm{d} x \\
& \quad+\int_{\Omega} \sum_{i, j=1}^{N} a_{i j}^{\varepsilon}\left(x, u_{\varepsilon}\right) D_{j} u_{\varepsilon} D_{i} u_{\varepsilon} h_{n}^{\prime}\left(u_{\varepsilon}\right) \xi_{\varepsilon} \mathrm{d} x \\
& \quad=\int_{\Omega_{1}^{\varepsilon} \cup \Omega_{2}^{\varepsilon}} F h_{n}\left(u_{\varepsilon}\right) \xi_{\varepsilon} \mathrm{d} x+\left.A_{\varepsilon} h_{n}\left(\left.u_{\varepsilon}\right|_{\tilde{\Gamma}_{1}^{\varepsilon} \cup \tilde{\Omega}^{\varepsilon} \cup \tilde{\Gamma}_{2}^{\varepsilon}}\right) \xi_{\varepsilon}\right|_{\tilde{\Gamma}_{1}^{\varepsilon} \cup \tilde{\Omega}^{\varepsilon} \cup \tilde{\Gamma}_{2}^{\varepsilon}} .
\end{aligned}
$$

Setting $M=4 k+h$, it is easy to see that $D \xi_{\varepsilon}=D T_{2 k}\left(u_{\varepsilon}-T_{h}\left(u_{\varepsilon}\right)+T_{k}\left(u_{\varepsilon}\right)-\right.$ $\left.T_{k}\left(\omega_{\varepsilon_{0}}\right)\right)=0$ as $\left|u_{\varepsilon}\right|>M$. Therefore for $n>M+1$, we have

$$
\begin{aligned}
& \int_{\Omega} \sum_{i, j=1}^{N} a_{i j}^{\varepsilon}\left(x, u_{\varepsilon}\right) D_{j} u_{\varepsilon} D_{i} \xi_{\varepsilon} h_{n}\left(u_{\varepsilon}\right) \mathrm{d} x \\
& \quad=\int_{\Omega} \sum_{i, j=1}^{N} a_{i j}^{\varepsilon}\left(x, T_{M}\left(u_{\varepsilon}\right)\right) D_{j} T_{M}\left(u_{\varepsilon}\right) D_{i} \xi_{\varepsilon} h_{n}\left(u_{\varepsilon}\right) \mathrm{d} x .
\end{aligned}
$$

By $\left(H_{5}\right),(4.15)$ and $(2.45)-(2.46)$, we obtain

$$
\begin{aligned}
\lim _{n \rightarrow \infty} & \int_{\Omega} \sum_{i, j=1}^{N} a_{i j}^{\varepsilon}\left(x, u_{\varepsilon}\right) D_{j} u_{\varepsilon} D_{i} \xi_{\varepsilon} h_{n}\left(u_{\varepsilon}\right) \mathrm{d} x \\
= & \int_{\Omega} \sum_{i, j=1}^{N} a_{i j}^{\varepsilon}\left(x, T_{M}\left(u_{\varepsilon}\right)\right) D_{j} T_{M}\left(u_{\varepsilon}\right) D_{i} \xi_{\varepsilon} \mathrm{d} x \\
\geq & \int_{\Omega} \sum_{i, j=1}^{N} a_{i j}^{\varepsilon}\left(x, T_{k}\left(u_{\varepsilon}\right)\right) D_{j} T_{k}\left(u_{\varepsilon}\right) D_{i}\left[T_{k}\left(u_{\varepsilon}\right)-T_{k}\left(\omega_{\varepsilon_{0}}\right)\right] \mathrm{d} x \\
& -\int_{\left\{x \in \Omega:\left|u_{\varepsilon}(x)\right|>k\right\}} \sum_{i, j=1}^{N}\left|a_{i j}^{\varepsilon}\left(x, T_{M}\left(u_{\varepsilon}\right)\right)\right|\left|D_{j} T_{M}\left(u_{\varepsilon}\right)\right|\left|D_{i} T_{k}\left(\omega_{\varepsilon_{0}}\right)\right| \mathrm{d} x .
\end{aligned}
$$

Sobolev imbedding theorem, trace theorem and (4.11) imply that

$$
\omega_{\varepsilon_{0}} \rightarrow u \quad \text { a.e } x \in \Omega, \quad \text { as } \varepsilon_{0} \rightarrow 0
$$

and

$$
\left.\omega_{\varepsilon_{0}}\right|_{\widetilde{\Gamma}_{1}^{\varepsilon} \cup \widetilde{\Omega}^{\varepsilon} \cup \widetilde{\Gamma}_{2}^{\varepsilon}}=\left.\left.\omega_{\varepsilon_{0}}\right|_{\widetilde{\Gamma}} \longrightarrow u\right|_{\widetilde{\Gamma}}, \quad \text { as } \varepsilon_{0} \rightarrow 0 .
$$

It follows from (4.11) and (4.12) that

$$
\lim _{\varepsilon_{0} \rightarrow 0} \lim _{\varepsilon \rightarrow 0} \int_{\left\{\left|u_{\varepsilon}\right|>k\right\}}\left|D_{i} T_{k}\left(\omega_{\varepsilon_{0}}\right)\right|^{2} \mathrm{~d} x=0 .
$$


For any fixed $h>0,\left(H_{6}\right)$ and (4.7) imply that $a_{i j}^{\varepsilon}\left(x, T_{M}\left(u_{\varepsilon}\right)\right) D_{j} T_{M}\left(u_{\varepsilon}\right)$ is bounded in $L^{2}(\Omega)$. Thus we can deduce that

$$
\lim _{\varepsilon_{0} \rightarrow 0} \lim _{\varepsilon \rightarrow 0} \int_{\left\{\left|u_{\varepsilon}\right|>k\right\}}\left|a_{i j}^{\varepsilon}\left(x, T_{M}\left(u_{\varepsilon}\right)\right)\right|\left|D_{j} T_{M}\left(u_{\varepsilon}\right)\right|\left|D_{i} T_{k}\left(\omega_{\varepsilon_{0}}\right)\right| \mathrm{d} x=0 .
$$

Obviously, we have

$$
\begin{aligned}
& \left|\int_{\Omega} \sum_{i, j=1}^{N} a_{i j}^{\varepsilon}\left(x, u_{\varepsilon}\right) D_{j} u_{\varepsilon} D_{i} u_{\varepsilon} h_{n}^{\prime}\left(u_{\varepsilon}\right) \xi_{\varepsilon} \mathrm{d} x\right| \\
& \quad \leq 2 k \int_{\left\{x \in \Omega: n \leq\left|u_{\varepsilon}(x)\right| \leq n+1\right\}} \sum_{i, j=1}^{N} a_{i j}^{\varepsilon}\left(x, u_{\varepsilon}\right) D_{j} u_{\varepsilon} D_{i} u_{\varepsilon} \mathrm{d} x .
\end{aligned}
$$

By (4.2) and (4.18), we see that

$$
\lim _{n \rightarrow \infty}\left|\int_{\Omega} \sum_{i, j=1}^{N} a_{i j}^{\varepsilon}\left(x, u_{\varepsilon}\right) D_{j} u_{\varepsilon} D_{i} u_{\varepsilon} h_{n}^{\prime}\left(u_{\varepsilon}\right) \xi_{\varepsilon} \mathrm{d} x\right|=0
$$

By Lebesgue's dominated convergence theorem, we have

$$
\lim _{h \rightarrow \infty} \lim _{\varepsilon_{0} \rightarrow 0} \lim _{\varepsilon \rightarrow 0} \lim _{n \rightarrow \infty} \int_{\Omega_{1}^{\varepsilon} \cup \Omega_{2}^{\varepsilon}} F h_{n}\left(u_{\varepsilon}\right) \xi_{\varepsilon} \mathrm{d} x=0 .
$$

According to $(2.45),(2.46),(4.9)$ and $(4.17)$, it is easy to get

$$
\left.\lim _{h \rightarrow \infty} \lim _{\varepsilon_{0} \rightarrow 0} \lim _{\varepsilon \rightarrow 0} \lim _{n \rightarrow \infty} A_{\varepsilon} h_{n}\left(\left.u_{\varepsilon}\right|_{\tilde{\Gamma}_{1}^{\varepsilon} \cup \tilde{\Omega}^{\varepsilon} \cup \tilde{\Gamma}_{2}^{\varepsilon}}\right) \xi_{\varepsilon}\right|_{\tilde{\Gamma}_{1}^{\varepsilon} \cup \tilde{\Omega}^{\varepsilon} \cup \tilde{\Gamma}_{2}^{\varepsilon}}=0 .
$$

Let $n$ tend to the infinity in (4.14). By the above relations, we get

$$
\varlimsup_{\varepsilon_{0} \rightarrow 0} \varlimsup_{\varepsilon \rightarrow 0} \int_{\Omega} \sum_{i, j=1}^{N} a_{i j}^{\varepsilon}\left(x, T_{k}\left(u_{\varepsilon}\right)\right) D_{j} T_{k}\left(u_{\varepsilon}\right) D_{i}\left[T_{k}\left(u_{\varepsilon}\right)-T_{k}\left(\omega_{\varepsilon_{0}}\right)\right] \mathrm{d} x=0 .
$$

For any given $k>0,(4.8)$ yields

$$
T_{k}\left(u_{\varepsilon}\right) \rightarrow T_{k}(u) \quad \text { weakly in } V .
$$

For any fixed $h>0$ and $k>0,\left(H_{6}\right)$ implies that $a_{i j}^{\varepsilon}\left(x, T_{k}\left(u_{\varepsilon}\right)\right) D_{j} T_{k}(u)$ is bounded in $L^{2}(\Omega)$. By $\left(H_{7}\right)$, it is easy to get as $\varepsilon \rightarrow 0$

$$
a_{i j}^{\varepsilon}\left(x, T_{k}\left(u_{\varepsilon}\right)\right) D_{j} T_{k}\left(\omega_{\varepsilon_{0}}\right) \rightarrow a_{i j}\left(x, T_{k}(u)\right) D_{j} T_{k}\left(\omega_{\varepsilon_{0}}\right) \quad \text { stronly in } L^{2}(\Omega) \text {. }
$$

By (4.12) and (4.20)-(4.21), we obtain

$$
\lim _{\varepsilon_{0} \rightarrow 0} \lim _{\varepsilon \rightarrow 0} \int_{\Omega} \sum_{i, j=1}^{N} a_{i j}^{\varepsilon}\left(x, T_{k}\left(u_{\varepsilon}\right)\right) D_{j} T_{k}\left(\omega_{\varepsilon_{0}}\right) D_{i}\left[T_{k}\left(u_{\varepsilon}\right)-T_{k}\left(\omega_{\varepsilon_{0}}\right)\right] \mathrm{d} x=0 .
$$

From (4.19) and (4.22), it follows

$$
\begin{aligned}
& \varlimsup_{\varepsilon_{0} \rightarrow 0} \varlimsup_{\varepsilon \rightarrow 0} \int_{\Omega} \sum_{i, j=1}^{N} a_{i j}^{\varepsilon}\left(x, T_{k}\left(u_{\varepsilon}\right)\right) D_{j}\left[T_{k}\left(u_{\varepsilon}\right)-T_{k}\left(\omega_{\varepsilon_{0}}\right)\right] D_{i}\left[T_{k}\left(u_{\varepsilon}\right)\right. \\
& \left.-T_{k}\left(\omega_{\varepsilon_{0}}\right)\right] \mathrm{d} x=0 .
\end{aligned}
$$


Assumption $\left(H_{5}\right)$ and (4.23) yield

$$
\varlimsup_{\varepsilon_{0} \rightarrow 0} \varlimsup_{\varepsilon \rightarrow 0}\left\|D T_{k}\left(u_{\varepsilon}\right)-D T_{k}\left(\omega_{\varepsilon_{0}}\right)\right\|_{L^{2}(\Omega)}=0 .
$$

Using (4.12) and (4.24), we obtain

$$
D T_{k}\left(u_{\varepsilon}\right) \rightarrow D T_{k}(u) \text { strongly in } L^{2}(\Omega), \quad \text { as } \varepsilon \rightarrow 0 .
$$

Thus, it follows from (4.25) that (4.10) holds.

Step 2: We will prove that $u$ satisfies (3.2).

By Lemma 4.4 and its proof, for any given $\eta \in V \cap L^{\infty}(\Omega)$, there is a sequence $\eta_{\varepsilon_{0}} \in V_{\varepsilon_{0}} \cap L^{\infty}(\Omega)$ such that

$$
\eta_{\varepsilon_{0}} \rightarrow \eta \text { strongly in } V \cap L^{\infty}(\Omega), \quad \text { as } \varepsilon_{0} \rightarrow 0 \text {. }
$$

Sobolev imbedding theorem and (4.26) yield

$$
\eta_{\varepsilon_{0}} \longrightarrow \eta \text { a.e in } \Omega, \quad \text { as } \varepsilon_{0} \rightarrow 0
$$

and

$$
\left.\eta_{\varepsilon_{0}}\right|_{\tilde{\Gamma}_{1} \cup \tilde{\Omega}_{\varepsilon} \cup \tilde{\Gamma}_{2}^{\varepsilon}}=\left.\left.\eta_{\varepsilon_{0}}\right|_{\tilde{\Gamma}} \rightarrow u\right|_{\tilde{\Gamma}}, \quad \text { as } \varepsilon_{0} \rightarrow 0
$$

For fixed $\varepsilon_{0}>0$ and any $\varepsilon$ with $0<\varepsilon<\varepsilon_{0}$, by (4.13) we have $\eta_{\varepsilon_{0}} \in$ $V_{\varepsilon} \cap L^{\infty}(\Omega)$. For any given $h \in W^{1, \infty}(R)$ with compact supp $h \subset[-L, L]$, let

$$
Q=\int_{\Omega} \sum_{i, j=1}^{N} a_{i j}^{\varepsilon}\left(x, u_{\varepsilon}\right) D_{j} u_{\varepsilon} D_{i} u_{\varepsilon} h^{\prime}\left(u_{\varepsilon}\right) \eta_{\varepsilon_{0}} \mathrm{~d} x .
$$

For any given $\widetilde{\Omega}$ such that $\widetilde{\Gamma} \subset \widetilde{\Omega} \subset \Omega$, we have

$$
\begin{aligned}
Q= & \int_{\Omega} \sum_{i, j=1}^{N} a_{i j}^{\varepsilon}\left(x, T_{L}\left(u_{\varepsilon}\right)\right) D_{j} T_{L}\left(u_{\varepsilon}\right) D_{i} T_{L}\left(u_{\varepsilon}\right) h^{\prime}\left(u_{\varepsilon}\right) \eta_{\varepsilon_{0}} \mathrm{~d} x \\
= & \int_{\Omega \backslash \widetilde{\Omega}} \sum_{i, j=1}^{N}\left[a_{i j}^{\varepsilon}\left(x, T_{L}\left(u_{\varepsilon}\right)\right)-a_{i j}\left(x, T_{L}\left(u_{\varepsilon}\right)\right)\right] D_{j} T_{L}\left(u_{\varepsilon}\right) D_{i} T_{L}\left(u_{\varepsilon}\right) h^{\prime}\left(u_{\varepsilon}\right) \eta_{\varepsilon_{0}} \mathrm{~d} x \\
& +\int_{\widetilde{\Omega}} \sum_{i, j=1}^{N} a_{i j}^{\varepsilon}\left(x, T_{L}\left(u_{\varepsilon}\right)\right) D_{j} T_{L}\left(u_{\varepsilon}\right) D_{i} T_{L}\left(u_{\varepsilon}\right) h^{\prime}\left(u_{\varepsilon}\right) \eta_{\varepsilon_{0}} \mathrm{~d} x \\
& +\int_{\Omega} \sum_{i, j=1}^{N} a_{i j}\left(x, T_{L}\left(u_{\varepsilon}\right)\right) D_{j} T_{L}\left(u_{\varepsilon}\right) D_{i} T_{L}\left(u_{\varepsilon}\right) h^{\prime}\left(u_{\varepsilon}\right) \eta_{\varepsilon_{0}} \mathrm{~d} x
\end{aligned}
$$




$$
\begin{aligned}
& -\int_{\widetilde{\Omega}} \sum_{i, j=1}^{N} a_{i j}\left(x, T_{L}\left(u_{\varepsilon}\right)\right) D_{j} T_{L}\left(u_{\varepsilon}\right) D_{i} T_{L}\left(u_{\varepsilon}\right) h^{\prime}\left(u_{\varepsilon}\right) \eta_{\varepsilon_{0}} \mathrm{~d} x \\
= & Q_{1}+Q_{2}+Q_{3}+Q_{4} .
\end{aligned}
$$

For any $\delta>0$, using $\left(H_{2}\right),\left(H_{6}\right),(4.10)$ and the absolute continuity of integral, we can take meas $\widetilde{\Omega}$ so small that as $0<\varepsilon<\varepsilon_{1}<\varepsilon_{0}$,

$$
\left|Q_{2}\right|+\left|Q_{4}\right|<\frac{\delta}{2}
$$

By the above choice of $\widetilde{\Omega}$, using (4.8), (4.25) and Vitali theorem, we get

$$
\begin{aligned}
\lim _{\varepsilon \longrightarrow 0} Q_{3} & =\int_{\Omega} \sum_{i, j=1}^{N} a_{i j}\left(x, T_{L}(u)\right) D_{j} T_{L}(u) D_{i} T_{L}(u) h^{\prime}(u) \eta_{\varepsilon_{0}} \mathrm{~d} x \\
& =\int_{\Omega} \sum_{i, j=1}^{N} a_{i j}(x, u) D_{j} u D_{i} u h^{\prime}(u) \eta_{\varepsilon_{0}} \mathrm{~d} x .
\end{aligned}
$$

By $\left(H_{7}\right)$ and (4.7), there exists $0<\varepsilon_{2} \leq \varepsilon_{1}$ such that for any $\varepsilon$ with $0<\varepsilon<\varepsilon_{2}$

$$
\left|Q_{1}\right|<\frac{\delta}{2}
$$

From(4.27)-(4.30), it follows

$$
\lim _{\varepsilon \longrightarrow 0} Q=\int_{\Omega} \sum_{i, j=1}^{N} a_{i j}(x, u) D_{j} u D_{i} u h^{\prime}(u) \eta_{\varepsilon_{0}} \mathrm{~d} x .
$$

Arguing as in the proof of (4.31), we have

$$
\begin{gathered}
\lim _{\varepsilon} \int_{\Omega} \sum_{i, j=1}^{N} a_{i j}^{\varepsilon}\left(x, u_{\varepsilon}\right) D_{j} u_{\varepsilon} D_{i} \eta_{\varepsilon_{0}} h\left(u_{\varepsilon}\right) \mathrm{d} x \\
=\int_{\Omega} \sum_{i, j=1}^{N} a_{i j}(x, u) D_{j} u D_{i} \eta_{\varepsilon_{0}} h(u) \mathrm{d} x .
\end{gathered}
$$

It is easy to see that

$$
\lim _{\varepsilon \rightarrow 0} \int_{\Omega_{1}^{\varepsilon} \cup \Omega_{2}^{\varepsilon}} F h\left(u_{\varepsilon}\right) \eta_{\varepsilon_{0}} \mathrm{~d} x=\int_{\Omega} F(x) h(u) \eta_{\varepsilon_{0}} \mathrm{~d} x
$$

and

$$
\left.\lim _{\varepsilon \rightarrow 0} A_{\varepsilon} h\left(\left.u_{\varepsilon}\right|_{\tilde{\Gamma}_{1}^{\varepsilon} \cup \tilde{\Omega}^{\varepsilon} \cup \tilde{\Gamma}_{2}^{\varepsilon}}\right) \eta_{\varepsilon_{0}}\right|_{\tilde{\Gamma}_{1}^{\varepsilon} \cup \tilde{\Omega}^{\varepsilon} \cup \tilde{\Gamma}_{2}^{\varepsilon}}=\left.A h\left(\left.u\right|_{\widetilde{\Gamma}}\right) \eta_{\varepsilon_{0}}\right|_{\widetilde{\Gamma}^{\prime}} .
$$

Taking $\xi=\eta_{\varepsilon_{0}}$ in (4.1) and let $\varepsilon \rightarrow 0,(4.31)-(4.34)$ yield

$$
\begin{aligned}
& \int_{\Omega} \sum_{i, j=1}^{N} a_{i j}(x, u) D_{j} u D_{i} \eta_{\varepsilon_{0}} h(u) \mathrm{d} x+\int_{\Omega} \sum_{i, j=1}^{N} a_{i j}(x, u) D_{j} u D_{i} u h^{\prime}(u) \eta_{\varepsilon_{0}} \mathrm{~d} x \\
& \quad=\int_{\Omega} F h(u) \eta_{\varepsilon_{0}} \mathrm{~d} x+\left.A h\left(\left.u\right|_{\tilde{\Gamma}}\right) \eta_{\varepsilon_{0}}\right|_{\tilde{\Gamma}}
\end{aligned}
$$


Taking $\varepsilon_{0} \rightarrow 0$ in (4.35), (4.26) and (4.35) imply that

$$
\begin{aligned}
& \int_{\Omega} \sum_{i, j=1}^{N} a_{i j}(x, u) D_{j} u D_{i} \eta h(u) \mathrm{d} x+\int_{\Omega} \sum_{i, j=1}^{N} a_{i j}(x, u) D_{j} u D_{i} u h^{\prime}(u) \eta \mathrm{d} x \\
& \quad=\int_{\Omega} F h(u) \eta \mathrm{d} x+\left.A h\left(\left.u\right|_{\tilde{\Gamma}}\right) \eta\right|_{\tilde{\Gamma}} .
\end{aligned}
$$

Step 3: We will prove that $u$ satisfies (3.3).

Let $\xi=T_{1}\left(u_{\varepsilon}-T_{k}\left(\omega_{\varepsilon_{0}}\right)\right)$ and $h=h_{n}$ in (4.1), where $\left\{\omega_{\varepsilon_{0}}\right\}$ is the sequence taken in step 1 . Then

$$
\begin{aligned}
\int_{\Omega} & \sum_{i, j=1}^{N} a_{i j}^{\varepsilon}\left(x, u_{\varepsilon}\right) D_{j} u_{\varepsilon} D_{i} T_{1}\left(u_{\varepsilon}-T_{k}\left(\omega_{\varepsilon_{0}}\right)\right) h_{n}\left(u_{\varepsilon}\right) \mathrm{d} x \\
& +\int_{\Omega} \sum_{i, j=1}^{N} a_{i j}^{\varepsilon}\left(x, u_{\varepsilon}\right) D_{j} u_{\varepsilon} D_{i} u_{\varepsilon} h_{n}^{\prime}\left(u_{\varepsilon}\right) T_{1}\left(u_{\varepsilon}-T_{k}\left(\omega_{\varepsilon_{0}}\right)\right) \mathrm{d} x \\
= & \int_{\Omega_{1}^{\varepsilon} \cup \Omega_{2}^{\varepsilon}} F h_{n}\left(u_{\varepsilon}\right) T_{1}\left(u_{\varepsilon}-T_{k}\left(\omega_{\varepsilon_{0}}\right)\right) \mathrm{d} x \\
& +A_{\varepsilon} h_{n}\left(\left.u_{\varepsilon}\right|_{\tilde{\Gamma}_{1}^{\varepsilon} \cup \tilde{\Omega}^{\varepsilon} \cup \tilde{\Gamma}_{2}^{\varepsilon}}\right) T_{1}\left(\left.u_{\varepsilon}\right|_{\tilde{\Gamma}_{1}^{\varepsilon} \cup \tilde{\Omega}^{\varepsilon} \cup \tilde{\Gamma}_{2}^{\varepsilon}}-T_{k}\left(\left.\omega_{\varepsilon_{0}}\right|_{\tilde{\Gamma}_{1}^{\varepsilon} \cup \tilde{\Omega}^{\varepsilon} \cup \tilde{\Gamma}_{2}^{\varepsilon}}\right)\right) .
\end{aligned}
$$

From (4.36), it follows

$$
\begin{aligned}
& \int_{\Omega} \sum_{i, j=1}^{N} a_{i j}^{\varepsilon}\left(x, u_{\varepsilon}\right) D_{j} u_{\varepsilon} D_{i} T_{1}\left(u_{\varepsilon}-T_{k}\left(\omega_{\varepsilon_{0}}\right)\right) h_{n}\left(u_{\varepsilon}\right) \mathrm{d} x \\
& \leq \int_{\Omega_{1}^{\varepsilon} \cup \Omega_{2}^{\varepsilon}} F h_{n}\left(u_{\varepsilon}\right) T_{1}\left(u_{\varepsilon}-T_{k}\left(\omega_{\varepsilon_{0}}\right)\right) \mathrm{d} x \\
& \quad+A_{\varepsilon} h_{n}\left(\left.u_{\varepsilon}\right|_{\tilde{\Gamma}_{1}^{\varepsilon} \cup \tilde{\Omega}^{\varepsilon} \cup \tilde{\Gamma}_{2}^{\varepsilon}}\right) T_{1}\left(\left.u_{\varepsilon}\right|_{\tilde{\Gamma}_{1}^{\varepsilon} \cup \tilde{\Omega}^{\varepsilon} \cup \tilde{\Gamma}_{2}^{\varepsilon}}-T_{k}\left(\left.\omega_{\varepsilon_{0}}\right|_{\tilde{\Gamma}_{1}^{\varepsilon} \cup \tilde{\Omega}^{\varepsilon} \cup \tilde{\Gamma}_{2}^{\varepsilon}}\right)\right) \\
& \quad+\int_{\left\{x \in \Omega: n \leq\left|u_{\varepsilon}\right| \leq n+1\right\}} \sum_{i, j=1}^{N} a_{i j}^{\varepsilon}\left(x, u_{\varepsilon}\right) D_{j} u_{\varepsilon} D_{i} u_{\varepsilon} \mathrm{d} x .
\end{aligned}
$$

Let $n$ tend to the infinity in (4.37). We get

$$
\begin{aligned}
& \int_{\Omega} \sum_{i, j=1}^{N} a_{i j}^{\varepsilon}\left(x, u_{\varepsilon}\right) D_{j} u_{\varepsilon} D_{i} T_{1}\left(u_{\varepsilon}-T_{k}\left(\omega_{\varepsilon_{0}}\right)\right) \mathrm{d} x \\
& \leq \int_{\Omega_{1}^{\varepsilon} \cup \Omega_{2}^{\varepsilon}} F T_{1}\left(u_{\varepsilon}-T_{k}\left(\omega_{\varepsilon_{0}}\right)\right) \mathrm{d} x+A_{\varepsilon} T_{1}\left(\left.u_{\varepsilon}\right|_{\tilde{\Gamma}_{1}^{\varepsilon} \cup \tilde{\Omega}^{\varepsilon} \cup \tilde{\Gamma}_{2}^{\varepsilon}}-T_{k}\left(\left.\omega_{\varepsilon_{0}}\right|_{\tilde{\Gamma}_{1}^{\varepsilon} \cup \tilde{\Omega}^{\varepsilon} \cup \tilde{\Gamma}_{2}^{\varepsilon}}\right)\right) .
\end{aligned}
$$


We can rewrite (4.38) as follows:

$$
\begin{aligned}
& \int_{\Omega} \sum_{i, j=1}^{N} a_{i j}^{\varepsilon}\left(x, T_{k+1}\left(u_{\varepsilon}\right)\right) D_{j} T_{k+1}\left(u_{\varepsilon}\right) \\
& \times\left[D_{i} T_{k+1}\left(u_{\varepsilon}\right)-D_{i} T_{k}\left(\omega_{\varepsilon_{0}}\right)\right] \chi_{\left\{\left|u_{\varepsilon}-T_{k}\left(\omega_{\varepsilon_{0}}\right)\right| \leq 1\right\}} \mathrm{d} x \\
& \leq \int_{\Omega_{1}^{\varepsilon} \cup \Omega_{2}^{\varepsilon}} F T_{1}\left(u_{\varepsilon}-T_{k}\left(\omega_{\varepsilon_{0}}\right)\right) \mathrm{d} x+A_{\varepsilon} T_{1}\left(\left.u_{\varepsilon}\right|_{\tilde{\Gamma}_{1}^{\varepsilon} \cup \tilde{\Omega}^{\varepsilon} \cup \tilde{\Gamma}_{2}^{\varepsilon}}-T_{k}\left(\left.\omega_{\varepsilon_{0}}\right|_{\tilde{\Gamma}_{1}^{\varepsilon} \cup \tilde{\Omega}^{\varepsilon} \cup \tilde{\Gamma}_{2}^{\varepsilon}}\right)\right) .
\end{aligned}
$$

Arguing as in the proof of (4.31), we can prove

$$
\begin{aligned}
\lim _{\varepsilon \longrightarrow 0} & \int_{\Omega} \sum_{i, j=1}^{N} a_{i j}^{\varepsilon}\left(x, T_{k+1}\left(u_{\varepsilon}\right)\right) D_{j} T_{k+1}\left(u_{\varepsilon}\right) \\
& \times\left[D_{i} T_{k+1}\left(u_{\varepsilon}\right)-D_{i} T_{k}\left(\omega_{\varepsilon_{0}}\right)\right] \chi_{\left\{\left|u_{\varepsilon}-T_{k}\left(\omega_{\varepsilon_{0}}\right)\right| \leq 1\right\}} \mathrm{d} x \\
= & \int_{\Omega} \sum_{i, j=1}^{N} a_{i j}\left(x, T_{k+1}(u)\right) D_{j} T_{k+1}(u) \\
& \times\left[D_{i} T_{k+1}(u)-D_{i} T_{k}\left(\omega_{\varepsilon_{0}}\right)\right] \chi_{\left\{\left|u-T_{k}\left(\omega_{\varepsilon_{0}}\right)\right| \leq 1\right\}} \mathrm{d} x .
\end{aligned}
$$

We can easily prove

$$
\lim _{\varepsilon \rightarrow 0} \int_{\Omega_{1}^{\varepsilon} \cup \Omega_{2}^{\varepsilon}} F T_{1}\left(u_{\varepsilon}-T_{k}\left(\omega_{\varepsilon_{0}}\right)\right) \mathrm{d} x=\int_{\Omega} F T_{1}\left(u-T_{k}\left(\omega_{\varepsilon_{0}}\right)\right) \mathrm{d} x
$$

and

$$
\lim _{\varepsilon \rightarrow 0} A_{\varepsilon} T_{1}\left(\left.u_{\varepsilon}\right|_{\tilde{\Gamma}_{1}^{\varepsilon \cup \Omega^{\varepsilon} \cup \tilde{\Gamma}_{2}^{\varepsilon}}}-T_{k}\left(\left.\omega_{\varepsilon_{0}}\right|_{\tilde{\Gamma}_{1}^{\varepsilon \cup \tilde{\Omega}^{\varepsilon} \cup \tilde{\Gamma}_{2}^{\varepsilon}}}\right)\right)=A T_{1}\left(\left.u\right|_{\tilde{\Gamma}}-T_{k}\left(\left.\omega_{\varepsilon_{0}}\right|_{\tilde{\Gamma}}\right)\right) .
$$

Thus, we obtain

$$
\begin{aligned}
& \int_{\Omega} \sum_{i, j=1}^{N} a_{i j}\left(x, T_{k+1}(u)\right) D_{j} T_{k+1}(u)\left[D_{i} T_{k+1}(u)-D_{i} T_{k}\left(\omega_{\varepsilon_{0}}\right)\right] \chi_{\left\{\left|u-T_{k}\left(\omega_{\varepsilon_{0}}\right)\right| \leq 1\right\}} \mathrm{d} x \\
& \quad \leq \int_{\Omega} F T_{1}\left(u-T_{k}\left(\omega_{\varepsilon_{0}}\right)\right) \mathrm{d} x+A T_{1}\left(\left.u\right|_{\tilde{\Gamma}}-T_{k}\left(\left.\omega_{\varepsilon_{0}}\right|_{\tilde{\Gamma}}\right)\right) .
\end{aligned}
$$

Let $\varepsilon_{0} \rightarrow 0$ in (4.39) and noting (4.11)-(4.13), we get

$$
\begin{aligned}
& \int_{\Omega} \sum_{i, j=1}^{N} a_{i j}\left(x, T_{k+1}(u)\right) D_{j} T_{k+1}(u)\left[D_{i} T_{k+1}(u)-D_{i} T_{k}(u)\right] \chi_{\left\{\left|u-T_{k}(u)\right| \leq 1\right\}} \mathrm{d} x \\
& \quad \leq \int_{\Omega} F T_{1}\left(u-T_{k}(u)\right) \mathrm{d} x+A T_{1}\left(\left.u\right|_{\tilde{\Gamma}}-T_{k}\left(\left.u\right|_{\tilde{\Gamma}}\right)\right) .
\end{aligned}
$$

We can rewrite (4.40) as

$$
\begin{aligned}
& \int_{\{x \in \Omega: k \leq|u(x)| \leq k+1\}} \sum_{i, j=1}^{N} a_{i j}(x, u) D_{j} u D_{i} u \mathrm{~d} x \\
& \leq \int_{\{x \in \Omega:|u(x)| \geq k\}}|F| \mathrm{d} x+A T_{1}\left(\left.u\right|_{\tilde{\Gamma}}-T_{k}\left(\left.u\right|_{\tilde{\Gamma}}\right)\right) .
\end{aligned}
$$


Hence, taking $k \rightarrow \infty$ in (4.41) we get

$$
\lim _{k \rightarrow \infty} \int_{\{x \in \Omega: k \leq|u(x)| \leq k+1\}} \sum_{i, j=1}^{N} a_{i j}(x, u) D_{j} u D_{i} u \mathrm{~d} x=0 .
$$

Thus we deduce that $u$ is a renormalized solution to problem $(P)$. Furthermore, by virtue of the uniqueness of renormalized solution to problem $(P)$, (4.8) holds for the whole sequence $\left\{u_{\varepsilon}\right\}$. This completes the proof of Theorem 4.3.

\section{References}

[1] Blanchard, D., Désir, F., Guibé, O.: Quasi-linear degenerate elliptic problems with $L^{1}$ data. Nonlinear Anal. 60, 557-587 (2005)

[2] Boccardo, L., Giachetti, D., Diaz, J.I., Murat, F.: Existence and regularity of renormalized solutions for some elliptic problems involving derivatives of nonlinear terms. J. Differ. Equ. 106(2), 215-237 (1993)

[3] Diperna, R.J., Lions, P.L.: On the Cauchy problem for the Boltzmann equations: global existence and weak stability. Ann. Math. 130(2), 321-366 (1989)

[4] Leone, C., Porretta, A.: Entropy solutions for nonlinear elliptic equations in $L^{1}$. Nonlinear Anal. 32, 325-334 (1998)

[5] Li, F.Q.: Limit behaviour of solutions to equivalued surface boundary value problem for P-Laplacian equations. Math. Methods Appl. Sci. 23, 723-733 (2000)

[6] Li, F.Q.: Limit behaviour of solutions to equivalued surface boundary value problem for P-Laplacian equations II. Math. Methods Appl. Sci. 24, 745$751(2001)$

[7] Li, F.Q.: Solvability of nonlinear elliptic boundary value problem with equivalued surface. Nonlinear Anal. 54, 995-1010 (2003)

[8] Li, T.T.: A class of non-local boundary value problems for particial differential equation and its applications in numerical analysis. J. Comput Appl. Math. 28, 49-62 (1989)

[9] Li, T.T., Tan, Y.J.: Mathematical problems and methods in resistivity well-loggings. Surv. Math. Ind. 5, 133-167 (1995)

[10] Li, T.T., Yan, J.H.: Limit behaviour of solutions to certain kinds of boundary value problems with equivalued surface. Asymptot. Analysis 21, 23-35 (1999)

[11] Li, T.T., Zheng, S.M., Tan, Y.J., Shen, W.X.: Boundary Value Problem With Equivalued Surface and Resistivity Well-Logging. Pitman Research Notes in Math Series, vol. 382. Longman, London (1998)

[12] Zhang, X., Li, F.Q.: Existence, uniqueness and limit behaviour of solutions to nonlinear boundary value problem with equivalued surface. Nonlinear Anal. 34, 525-536 (1998) 
[13] Zeidler, E.: Nonlinear Fountional Analysis and Its Applications. II/B: Nonlinear Monotone Operators. Springer, New York (1990)

F. Li, W. Zou

Department of Applied Mathematics,

Dalian University of Technology,

Dalian 116024, China

e-mail: fqli@dlut.edu.cn

W. Zou

e-mail: zwl267@yahoo.com.cn

Received: 29 September 2007.

Revised: 12 May 2009.

Accepted: 21 May 2010. 